\title{
Check list of fishes of the Gulf of Mannar ecosystem, Tamil Nadu, India
}

\author{
K. K. Joshi, Miriam Paul Sreeram, P. U. Zacharia, E. M. Abdussamad, Molly Varghese, 0. M. M. \\ J. Mohammed Habeeb', K. Jayabalan', K. P. Kanthan', K. Kannan', K. M. Sreekumar, Gimy \\ George and M. S. Varsha \\ ICAR-Central Marine Fisheries Research Institute, P. B. No.1603, Kochi - 682 018, Kerala, India. \\ 1Tuticorin Research Centre of Central Marine Fisheries Research Institute, Tuticorin - 628 001, Tamil Nadu, India. \\ *Correspondence e-mail: joshyguru@gmail.com
}

\begin{abstract}
Gulf of Mannar Ecosystem (GOME) covers an area spread over Rameswaram and Kanyakumari for about $19000 \mathrm{~km}^{2}$ and lies between $78^{\circ} 11^{\prime} \mathrm{E}$ and $79^{\circ} 15^{\prime} \mathrm{E}$ longitude and $8^{\circ} 49^{\prime} \mathrm{N}$ and $9^{\circ} 15^{\prime} \mathrm{N}$ latitude. The 21 coral islands form a network of habitats for different kinds of fishes and marine organisms. Fish samples were collected during April 2005 to March 2010 from different centers viz., Vembar, Tharuvaikulam, Vellapatti, Therespuram, Tuticorin, Alangarathattu, Pazhaykayal, Punnakayal, Kayalpattinam, Veerapandiapattinam, Thiruchendur and Manappad of Gulf of Mannar ecosystem. The present check list was the first attempt to produce a systematic list of fishes found in the Gulf of Mannar ecosystem. It includes 476 genera and 1182 species in 144 families and 39 orders, of which 628 species have been recorded in the present study.
\end{abstract}

Key words: Marine fishes, marine protected area, coral islands, elasmobranchs, teleosts, deep sea fishes.

\section{Introduction}

India is blessed with a vast region of coral reefs and mangroves and these regions support very rich fauna of flora and constitute rich biodiversity of marine organisms. Gulf of Mannar Ecosystem (GOME) covers an area spread over Rameswaram and Kanyakumari to about $19,000 \mathrm{~km}^{2}$. GOME lies between $78^{\circ} 11^{\prime} 00^{\prime \prime} \mathrm{E}$ and $79^{\circ} 15^{\prime} 00^{\prime \prime} \mathrm{E}$ longitude and $8^{\circ} 49^{\prime} 00^{\prime \prime} \mathrm{N}$ and $9^{\circ} 15^{\prime} 00^{\prime \prime} \mathrm{N}$ latitude. The GOME has within its limits the Gulf of Mannar Biosphere Reserve (GOMBR) which was declared in 1989 as a protected area with an area of $10,500 \mathrm{~km}^{2}$ and a Gulf of Mannar National Park (GOMNP). The GOMNP included 21 Islands and surrounding coral reefs with an area of $560 \mathrm{~km}^{2}$.

Fisheries is one of the most important renewable resources and provide livelihood for thousands of fishermen families residing in and around the coastal villages of the GOMBR. With increasing fishing pressure and conservation of the resources, the only option left for the sustainability of fisheries is their rational management. Proper management and conservation is possible with a thorough knowledge of the exact species identification and systematic list of the fish stocks present in the GOME. 
GOME is known for its pearl oyster fisheries and chank fisheries since 1900 (Hornell, 1922). During 1975-1985 surveys conducted in the pearl oyster beds (Paars) revealed the decline in the population of pearl oyster. Several reasons were attributed to the decline of pearl oyster fishery in GOME such as shifting of sand by bottom currents caused by the southwest monsoon, natural enemies like fishes of Balistidae, Lethrinus, Serranus, Tetradon, Rhinoptera javanica and other groups like boring molluscs, sponges, starfishes and crabs. Besides the over fishing, and imbalance in male and female ratios in the growth of the population in the pearl oyster beds also causes for the decline of the resources (Herdman, 1903; Hornell, 1916; Devanesan and Chidambaram, 1956; Mahadevan and Nayar, 1973). The shift from pearl oyster fisheries to fin fish resources was gradual and got momentum when the mechanization and motorization of crafts and gears occurred during the five year plan period of India. Diversity of fin fishes of GOM areas is high and inventorying of the resources is important in the context of biodiversity conservation and sustainable utilization of the resources.

The 21 coral islands starting from north most Pamban Island to Kanayakumari forms a network of habitats for different kinds of fishes both resident and migratory. Hare Island is the biggest island and is about seven $\mathrm{km}$ from Mandapam Camp having an area of 160 hectares and the smallest islands are Manoliputti Island, Pulivinchalli Island and Valimunai Island. Three rivers falling into GOM are Vembar, Thamaraparniyar and Vaipar forming estuaries in the GOM area. Important types of ecosystem are seagrass, seaweed, coral reefs and other habitats like rocky shore, sandy beach, muddy beach and mudflats. Due to the peculiarities of ecosystem and protective nature of the habitats several species of fishes undertake breeding migration to GOM area. Different species include pelagic, benthic, benthopelagic, deep sea, estuarine, brackish water, migratory and cave dwelling forms.

First report of the finfish species from GOM was during 1964 and contains a list of 265 fishes and the first attempt to bring out a checklist of fishes of GOM resulted in cataloging of about 300 species of fishes from GOM (Murty, 1969). In the past several attempts by different workers to study the fin fishes of GOME resulted in either listing of a group of fishes or fishes from a particular area or report of new records of a single species from this area (James, 1967; Thomas, 1969; Venkataramani \& Ramaiyan, 1977; Williams \& Venkataramani, 1978; James, 1985; Venkataramani et al., 1995; Varghese, 2011; Varghese et al., 2011; Zacharia et al.,

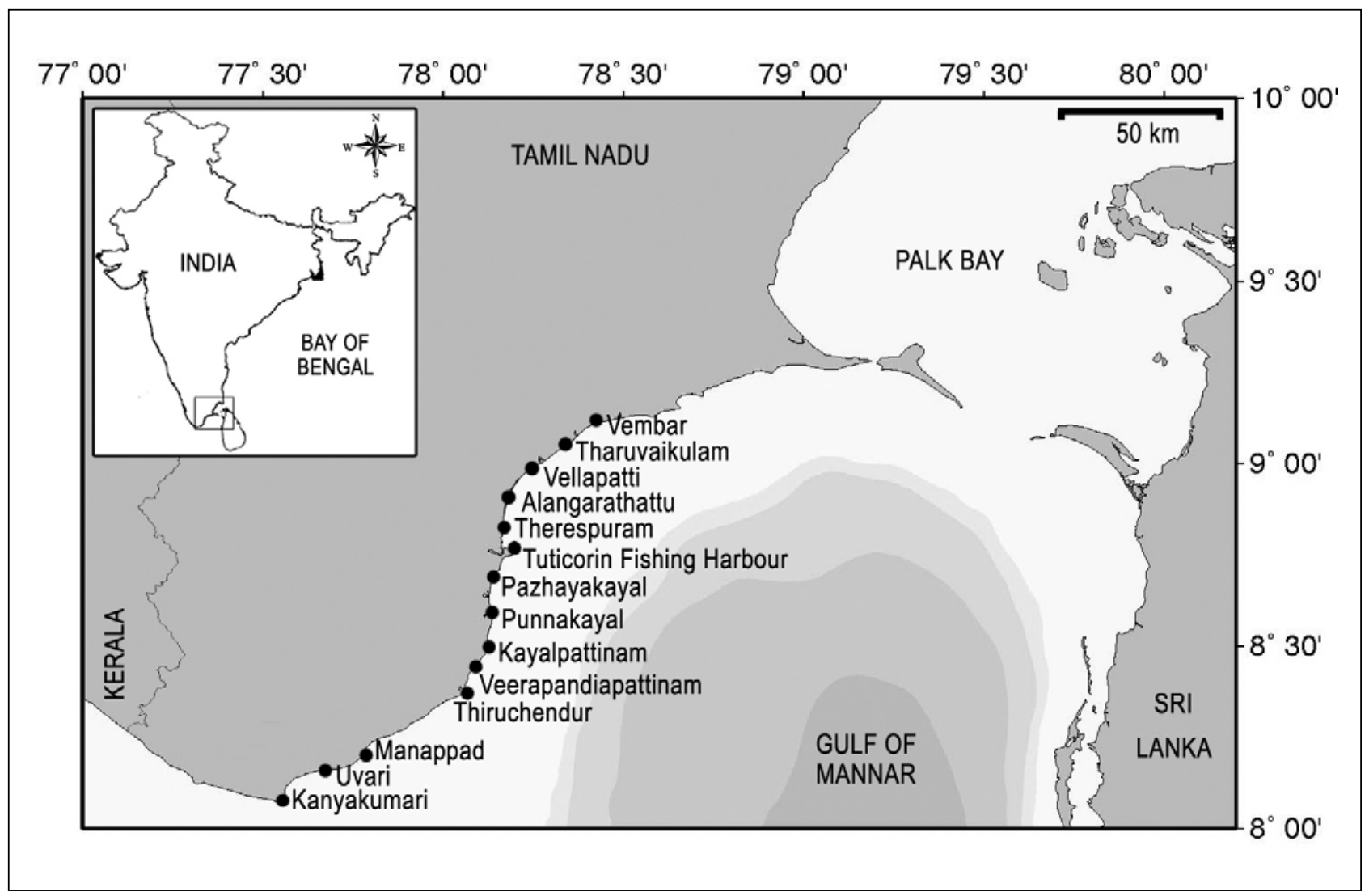

Fig. 1. Map showing study area of the Gulf of Mannar Ecosystem, India 
2011; Joshi et al., 2012b, Abraham et al., 2011; Manisseri et al., 2012; Zacharia and Kannan, 2012). Although these works added to the fish diversity of the GOM, a totality of species inventory is lacking and is causing problems for the conservation and management of this very important Marine Biosphere Reserve of the first of this kind in Tamil Nadu, India. In order to gather all the information, we have utilized all the taxonomical publications, reports, checked Eschmeyer (1998) and searched Catalog of Fishes (Eshmeyer and Fricke, 2015), Fishbase (Froese and Pauly, 2015) and other sites. The present check list was the first attempt to produce a systematic list of all the 1,182 species of fishes found in the Gulf of Mannar Ecosystem and adjacent areas of the Bay of Bengal. The ability to address the biodiversity utilization and conservation largely depends on the knowledge of the taxonomy of the flora and fauna constituting the biodiversity.

\section{Material and methods}

\section{Sample collection}

Fish samples were collected during April 2005 to March 2010. Sampling was conducted two to three consecutive days in a week from different centres like Vembar, Tharuvaikulam, Vellapatti, Therespuram, Tuticorin Harbour, Alangarathattu, Pazhaykayal, Punnakayal, Kayalpattinam, Veerapandiapattinam, Thiruchendur and Manappad of Gulf of Mannar Ecosystem (Fig. 1). Different types of gears were used for catching fish species from the GOME. Large meshed gillnets were used for catching species like tuna, seer fish, barracuda, and wolf herring. Small meshed gillnets were used for catching other types of species like sardine, mackerel, small carangids and miscellaneous species. Trawl nets were used for the exclusive fishery of different types and sizes of species from GOME. Gillnets of different names such as Nanduvalai, Chalavalai, Mayavalai, and Trammel net were also used to collect different varieties of marine organisms. Specialized trawl net "Thallumadi" was employed for catching fishes associated with seagrass beds and coral reef area where conventional trawl nets cannot operate smoothly. Shore seine of two types such as bigger nets (Karavalai) and smaller nets (Olavalai) were used in the near shore area to collect different species. Long lines were used to collect large pelagics from deeper areas. Hook and lines were used for catching species from the rocky shore areas and deeper waters. Besides, cast nets and traps were also used in certain places to collect fish samples. A total of 1,182 species of fishes was collected in the present study.

\section{Species Identification}

Identification of species was confirmed using standard Monographs and Fish Identification books (FAO Fish Identification sheets, Species Catalogue, Smith's Sea fishes).
In some cases photographs were sent to world authorities and the identification was confirmed. The classification as described by Nelson (2006) was followed. Nomenclature and taxonomical details were taken from the Catalog of Fishes (Eschmeyer, 1998). The present checklist contains a total of 1,182 species of fishes occuring in the GOM. The distributional details of some of the species which were not recorded in the present study was taken from the past studies (Day, 1878; Munro, 1955; Carpenter and Niem, 1998).

Species identification and systematic position of the fishes was done primarily using monographs and identification manuals (Cuvier and Valenciennes, 1828; Gunther, 18591970; Day, 1878; Day, 1888; Hamilton, 1822; Weber and deBeaufort, 1913-1940; Munro, 1955; Fischer and Whitehead, 1974; Jones and Kumaran, 1980; Talwar and Kacker, 1984; Smith and Heemstra, 1986; Talwar and Jhingran, 1991; Allen et al., 1997; Carpenter and Niem, 1998; Eschmeyer, 1998). The taxonomic reviews and recent publications of Chen et al. (1994: Anguilliformes); Roberts and Vidthayanon (1991: Siluriformes); Becker (1968: Myctophids); Kawaguchi and Shimizu (1978: Myctophidae); Kotlyar (1987; 1996: Bercyformes); Cressey (1981: Synodontidae); Randall and Greenfield (1996: Holocentridae); Markle and Olney (1990: Carapidae); Heemstra (1980: Zeidae); Dawson (1985: Pipe fishes); Lourie et al. (1999: Sea horses); Imamura (1996: Platycephalidae); Knapp (1996: Platycephalidae); Anderson (1970: Lutjanidae); Heemstra and Randall (1993: Serranidae); Katayama (1960; Serranidae); Randall and Baldwin (1997: Serranidae); Randall and Heemstra (1991: Serranidae); Randall and McCosker (1992: Serranidae); Gon (1993: Apogonidae); Randall et al. (1985: Apogonidae); McKay (1992: Sillaginidae); Dooley (1978: Branchiostegidae and Malacanthidae); Randall et al. (1987: Pinjalo); Allen (1985: Lutjanidae); Carpenter (1987; 1988: Caesionidae), Carpenter and Allen (1989: Lethrinidae); Russell (1990: Nemipteridae); Trewavas (1977: Sciaenidae); Lal Mohan (1981: Sciaenidae); Thomas (1969: Goat fishes); James (1985: Silverbellies); Abraham et al. (2011:Silverbellies); James (1967: Ribbon fishes); Allen (1979: Butterfly and Angelfishes); Vari (1978: Teraponidae); Senon (1988: Mugilidae); Thomson (1997: Mugilidae); Allen (1991: Damsel fishes); Randall (1982: Hologymnosus); Bellwood (1994: Scaridae); Choat and Randall (1986: Scaridae); Starnes (1988: Priacanthidae); Springer (1971: Blennidae); Springer and William (1994: Blennidae); Hoese and Larson (1994: Valencienna); Murdy and Hoeses (1985: Gobiidae); Murdy (1989: Gobiidae); Kishimoto et al. (1988: Platax); Woodland (1990: Siganidae); Lin and Shao (1987: Sphyraenidae); Nakamura and Parin (1993: Snake Mackerel); Collette and Nauen (1983: Scombridae); Menon (1977: Cynoglossidae); Munroe and Marsh (1997: Symphurus); Leis (1978: Diodontidae); Sparks and Chakrabarty, 2007); Chakrabarty and Sparks (2007; 2008: Silverbellies); 
Table 1. List of fish species occurring in the Gulf of Mannar Ecosystem, Tamil Nadu, India.

\begin{tabular}{|c|c|}
\hline Group & Name \\
\hline Class & ELASMOBRANCHII \\
\hline Sub-class & HOLOCEPHALI \\
\hline Order & CHIMAERIFORMES \\
\hline Family & Chimaeridae (shortnose chimeras or ratfishes) \\
\hline 1 & Hydrolagus africanus (Gilchrist, 1922) \\
\hline Family & Rhinochimaeridae (longnose chimeras) \\
\hline 2 & Neoharriotta pinnata (Schnakenbeck, 1931) \\
\hline 3 & Rhinochimaera pacifica (Mitsukuri, 1895) \\
\hline Sub-class & ELASMOBRANCHII \\
\hline Sub-division & SELACHII \\
\hline Order & HEXANCHIFORMES \\
\hline Family & Hexanchidae (cow sharks) \\
\hline 4 & Hexanchus griseus (Bonnaterre, 1788) \\
\hline Order & HETERODONTIFORMES \\
\hline Family & Heterodontidae (horned sharks) \\
\hline 5 & Heterodontus zebra (Gray, 1831) \\
\hline Order & ORECTOLOBIFORMES \\
\hline Family & Parascyllidae (collard carpet sharks) \\
\hline 6 & Cirrhoscyllium expolitum Smith \& Radcliffe, 1913 \\
\hline Family & Hemiscylliidae (bamboo sharks) \\
\hline 7 & Chiloscyllium indicum (Gmelin, 1789) \\
\hline 8 & Chiloscyllium griseum Muller \& Henle, 1838 \\
\hline Family & Stegostomatidae (zebra sharks) \\
\hline 9 & Stegostoma fasciatum (Hermann, 1783) \\
\hline Family & Ginglymostomatidae (nurse sharks) \\
\hline 10 & Nebrius ferrugineus (Lesson, 1831) \\
\hline Family & Rhincodontidae (whale sharks) \\
\hline
\end{tabular}

Orde

Family

Rhincodon typus Smith, 1828

LAMNIFORMES

\begin{tabular}{ll}
\hline 12 & Alopias pelagicus Nakamura, 1935 \\
\hline 13 & Alopias superciliosus Lowe, 1841 \\
\hline 14 & Alopius vulpinus (Bonnaterre, 1788) \\
\hline Family & Lamnidae (mackerel sharks) \\
\hline 15 & Lamna nasus (Springer, 1973) \\
\hline 16 & Carcharodon carcharias (Linnaeus, 1758) \\
\hline Family & Pseudocarcharhinidae (crocodile sharks) \\
\hline 17 & Pseudocarcharias kamoharai (Matsubara, 1936) \\
\hline Order & Isurus oxyrinchus Rafinesque, 1850 \\
\hline Family & CARCHARHINIFORMES \\
\hline 19 & Carcharhinidae (gray sharks) \\
\hline 20 & Carcharhinus altimus (Springer, 1950) \\
\hline 21 & Carcharhinus amboinensis (Muller \& Henle, 1839) \\
\hline 22 & Carcharhinus amblyrhynchoides (Whitely, 1934) \\
\hline 23 & Carcharhinus amblyrhynchos (Bleeker, 1856) \\
\hline 24 & Carcharhinus brevipinna (Muller \& Henle, 1841) \\
\hline
\end{tabular}

\begin{tabular}{|c|c|}
\hline 25 & Carcharhinus falciformis (Muller \& Henle, 1841) \\
\hline 26 & Carcharhinus hemiodon (Muller \& Henle, 1839) \\
\hline 27 & Carcharhinus leucas (Valenciennes, 1839) \\
\hline 28 & Carcharhinus limbatus (Muller \& Henle, 1839) \\
\hline 29 & Carcharhinus longimanus (Poey, 1861) \\
\hline 30 & Carcharhinus macloti (Muller \& Henle, 1839) \\
\hline 31 & Carcharhinus melanopterus (Quoy \& Gaimard, 1824) \\
\hline 32 & Carcharhinus obscurus (Lesueur, 1818) \\
\hline 33 & Carcharhinus plumbeus (Nardo, 1827) \\
\hline 34 & Carcharhinus sealei (Pietschmann, 1916) \\
\hline 35 & Carcharhinus sorrah (Muller \& Henle, 1839) \\
\hline 36 & Galeocerdo cuvieri (Lesuer, 1818) \\
\hline 37 & Lamiopsis temmincki (Muller \& Henle, 1839) \\
\hline 38 & Loxodon macrorhynus (Muller \& Henle, 1839) \\
\hline 39 & Negaprion acutidens (Rüppell, 1837) \\
\hline 40 & Prionace glauca (Linnaeus, 1758) \\
\hline 41 & Rhizoprionodon acutus (Rüppell, 1837) \\
\hline 42 & Rhizoprionodon oligolinx Springer, 1964 \\
\hline 43 & Scoliodon laticaudus (Muller \& Henle, 1839) \\
\hline 44 & Triaenodon obesus (Rüppell, 1837) \\
\hline Family & Sphyrnidae (hammerheaded shark) \\
\hline 45 & Eusphyra blochii (Cuvier, 1816) \\
\hline 46 & Sphyrna lewini (Griffith \& Smith, 1834) \\
\hline 47 & Sphyrna mokarran (Rüppell, 1837) \\
\hline 48 & Sphyrna zygaena (Linnaeus, 1758) \\
\hline Order & ECHINORHINIFORMES \\
\hline Family & Echinorhinidae (bramble shark) \\
\hline 49 & Echinorhinus brucus (Bonnaterre, 1788) \\
\hline Order & SQUALIFORMES \\
\hline Family & Squalidae (dogfishes) \\
\hline 50 & Squalus acanthias Linnaeus, 1758 \\
\hline 51 & Squalus blainville (Risso, 1827) \\
\hline Family & Centrophoridae (gulper sharks) \\
\hline 52 & Centrophorus granulosus (Bloch \& Schneider, 1801) \\
\hline 53 & Centrophorus uyato (Rafinesque, 1810) \\
\hline 54 & Centrophorus moluccensis (Bleeker, 1860) \\
\hline Sub-division & BATOIDEA \\
\hline Order & PRISTIFORMES \\
\hline Family & Pristidae (swordfishes) \\
\hline 55 & Anoxypristis cuspidata (Latham, 1794) \\
\hline 56 & Pristis microdon Latham, 1794 \\
\hline 57 & Pristis pectinata Latham, 1794 \\
\hline 58 & Pristis zijsron Bleeker, 1851 \\
\hline Order & TORPEDINIFORMES \\
\hline Family & Torpedinidae (electric rays) \\
\hline 59 & Narcine brunnea Annandale, 1909 \\
\hline 60 & Narcine indica Henle, 1834 \\
\hline 61 & Narcine timlei (Bloch \& Schneider, 1801) \\
\hline 62 & Narke dipterygia (Bloch \& Schneider,1801) \\
\hline 63 & Torpedo marmorata (Risso, 1810) \\
\hline
\end{tabular}




\begin{tabular}{|c|c|c|c|}
\hline Order & RAJIFORMES & Order & ALBULIFORMES \\
\hline Family & Rhinobatidae (guitarfishes) & Sub order & Albulidae \\
\hline 64 & Rhinobatos granulatus (Cuvier, 1829) & Family & Albulidae (bonefishes) \\
\hline 65 & Rhinobatos variegatus Nair \& Lal Mohan, 1973 & 103 & Albula vulpes (Linnaeus, 1758) \\
\hline 66 & Rhinobatos annandalei (Norman, 1926) & 104 & Albula neoguinaica Cuvier \& Valenciennes, 1847 \\
\hline 67 & Rhinobatos obtusus (Muller \& Henle, 1841) & Order & ANGUILLIFORMES \\
\hline 68 & Rhina ancylostoma Bloch \& Schneider, 1801 & Sub order & Anguilloidei \\
\hline 69 & Rhyncobatus djiddensis (Forsskål, 1775) & Family & Anguillidae (freshwater eels) \\
\hline Family & Dasyatidae (sting rays) & 105 & Anguilla bicolor McCelland, 1844 \\
\hline 70 & Dasyatis kuhli (Muller \& Henle, 1841) & 106 & Anguilla nebulosa McClelland, 1844 \\
\hline 71 & Dasyatis microps (Annandale, 1908) & 107 & Anguilla bengalensis (Gray, 1831) \\
\hline 72 & Dasyatis sephen (Forsskål, 1775) & Sub order & Muraenoidei \\
\hline 73 & Dasyatis zugei (Burger, 1841) & Family & Muraenidae (morays) \\
\hline 74 & Himantura imbricata (Bloch \& Schneider, 1801) & 108 & Echidna nebulosa (Ahl, 1789) \\
\hline 75 & Himantura bleekeri (Blyth, 1860) & 109 & Echidna zebra (Shaw, 1797) \\
\hline 76 & Himantura gerrardi (Grey, 1851) & 110 & Gymnothorax javanicus (Bleeker, 1859) \\
\hline 77 & Himantura jenkinsii (Annandale, 1909) & 111 & Gymnothorax favagineus Bloch \& Schneider, 1801 \\
\hline 78 & Himantura marginata (Blyth, 1860) & 112 & Gymnothorax flavimarginatus (Rüppell, 1830) \\
\hline 79 & Himantura uarnak (Gmelin, 1789) & 113 & Gymnothorax meleagris (Shaw, 1795) \\
\hline$\frac{80}{81}$ & $\begin{array}{l}\text { Himantura walga (Muller \& Henle, 1841) } \\
\text { Himantura undulata (Bleeker, 1852) }\end{array}$ & 114 & Gymnothorax octaviana (Myers \& Wade, 1941) \\
\hline 82 & Pteroplatytrygon violacea (Bonaparte, 1832) & 115 & Gymnothorax pictus (Ahl, 1789) \\
\hline 83 & Taeniura lymma (Forsskål, 1775) & 116 & Gymnothorax rueppelliae (McCelland, 1844) \\
\hline 84 & Taeniura meyeni Muller \& Henle, 1841 & 117 & Gymnothorax thyrsoideus (Richardson, 1845) \\
\hline 85 & Urogymnus granulatus (Macleay, 1883) & 118 & Gymnothorax undulatus (Lacepède, 1803) \\
\hline 86 & Urogymnus asperrimus (Bloch \& Schneider, 1801) & 119 & Strophidon sathete (Hamilton, 1822) \\
\hline Family & Gymnuridae (butterfly rays) & 120 & Thyrosidea macrura (Bleeker, 1854) \\
\hline 87 & Gymnura japonica (Temminck \& Schlegel, 1850) & 121 & Uropterygius concolor Rüppell, 1838 \\
\hline 88 & Gymnura poecilura (Shaw, 1804) & Sub order & Congroidei \\
\hline 89 & Gymnura micrura (Bloch \& Schneider, 1801) & Family & Congridae (conger eels, garden eels) \\
\hline Family & Myliobatidae (angle, devil, cownose rays) & 122 & Ariosoma anago (Temmink \& Schlegel, 1846) \\
\hline 90 & Aetobatus flagellum (Bloch \& Schneider, 1801) & 123 & Ariosoma anagoides (Bleeker, 1853) \\
\hline 91 & Aetobatus narinari (Euphrasen, 1790) & 124 & Ariosoma marginatum (Vaillant \& Sauvage, 1875) \\
\hline 93 & Manta birostris (Walbaum, 1792) & 125 & Conger cinereus Rüppell, 1871 \\
\hline 94 & Mobula diabolus (Shaw, 1804) & 126 & Conger japonicus Bleeker, 1879 \\
\hline 95 & Mobula mobular (Bonnaterre, 1788) & 127 & Paraconger macrops (Günther, 1870) \\
\hline 96 & Mobula thurstoni (Lloyd, 1908) & 128 & Paraconger notialis Kanazawa, 1961 \\
\hline 97 & Myliobatus maculatus Gray, 1834 & 129 & Rhynchoconger nitens (Jordan \& Bollman, 1890) \\
\hline 98 & Rhinoptera jayakari Boulenger, 1895 & 130 & Uroconger lepturus (Richardson, 1845) \\
\hline 99 & Rhinoptera javanica (Muller \& Henle, 1841) & Family & Ophichthidae (snake eels) \\
\hline Class & ACTINOPTERYGII (OSTEICHTHYES) & 131 & Caecula pterygera Vahl, 1794 \\
\hline Sub-class & NEOPTERYGII & 132 & Lamnostoma orientalis (McClelland, 1844) \\
\hline Division & TELEOSTEI & 133 & Pisodonophis boro (Hamilton, 1822) \\
\hline Order & ELOPIFORMES & 134 & Pisodonophis cancrivorus (Richardson, 1848) \\
\hline Family & Elopidae (ten pounders) & Family & Muraenesocidae (pike congers) \\
\hline$\frac{100}{101}$ & $\begin{array}{l}\text { Elops saurus Linnaeus, 1/66 } \\
\text { Elops machnata (Forsskål, 1775) }\end{array}$ & 135 & Muraenesox cinereus (Forsskål, 1775) \\
\hline Family & Megalopidae (tarpons) & 136 & Congresox talabanoides (Bleeker, 1852) \\
\hline 102 & Megalops cyprinoides (Broussonet, 1782) & 137 & Congresox talabon (Cuvier, 1829) \\
\hline
\end{tabular}




\begin{tabular}{|c|c|c|c|}
\hline Order & CLUPEIFORMES & 182 & Raconda russeliana Gray, 1831 \\
\hline Sub order & Clupeoidei & 183 & Sardinella albella (Valenciennes, 1847) \\
\hline Family & Pristigasteridae (pristigasterids) & 184 & Sardinella brachysoma (Bleeker, 1852) \\
\hline 138 & Hilsa ilisha (Hamilton, 1822) & 185 & Sardinella dayi Regan,1917 \\
\hline 139 & Hilsa keele (Cuvier, 1829) & 186 & Sardinella fimbriata (Valenciennes, 1847) \\
\hline 140 & Hilsa toli (Valenciennes, 1847) & 187 & Sardinella gibbosa (Bleeker, 1849) \\
\hline 141 & Ilisha elongata Bennett, 1830 & 188 & Sardinella longiceps Valenciennes, 1847 \\
\hline 142 & Ilisha melastoma (Bloch \& Schneider, 1801) & 189 & Sardinella melanura (Cuvier, 1829) \\
\hline 143 & Ilisha sirishae Seshagiri Rao, 1975 & 190 & Sardinella sirm (Walbaum, 1729) \\
\hline 144 & Ilisha striatula Wongratana, 1983 & 191 & Spratelloides malabaricus (Schlegel \& Temminck, 1846) \\
\hline 145 & Pellona dayi Wongratana, 1983 & 192 & Spratelloides gracilis (Schlegel \& Temminck, 1846) \\
\hline Family & Engraulidae (anchovies) & 193 & Spratelloides delicatulus (Bennet, 1832) \\
\hline 146 & Coilia borneensis Bleeker, 1852 & 194 & Tenualosa toli (Valenciennes, 1847) \\
\hline 147 & Coilia dussumieri Valenciennes, 1848 & Order & GONORYNCHIFORMES \\
\hline 148 & Coilia neglecta Whitehead, 1968 & Sub order & Chanoidei \\
\hline 149 & Encrasicholina punctifer Fowler, 1938 & Family & Chanidae (milkfishes) \\
\hline 150 & Encrasicholina devisi (Whitley, 1940) & 195 & Chanos chanos (Forsskål, 1775) \\
\hline 151 & Encrasicholina heteroloba (Rüppell, 1837) & Order & SILURIFORMES \\
\hline 152 & Engraulis macrops Kishinouye, 1911 & Family & Plotosidae (eeltail catfishes) \\
\hline 153 & Setipinna melanochir (Bleeker, 1849) & 196 & Plotosus canius Hamilton, 1822 \\
\hline 154 & Setipinna taty (Valenciennes, 1848) & 197 & Plotosus abbreviatus Boulenger, 1895 \\
\hline 155 & Setipinna tenuifilis (Valenciennes, 1848) & 198 & Plotosus lineatus (Thrunberg, 1787) \\
\hline 156 & Stolephorus dubiosus Wongratana, 1983 & 199 & Plotosus limbatus Valenciennes, 1840 \\
\hline 157 & Stolephorus commersonnii Lacepède, 1803 & Family & Ariidae (sea catfishes) \\
\hline 158 & Stolephorus devisi (Whitley, 1940) & 200 & Arius jella Day, 1877 \\
\hline 159 & Stolephorus indicus (van Hasselt, 1823) & 201 & Arius arius (Hamilton, 1822) \\
\hline 160 & Stolephorus waitei Jordan \& Seale, 1926 & 202 & Arius caelatus Valenciennes, 1840 \\
\hline 161 & Thryssa hamiltonii (Gray, 1835) & 203 & Arius dussumieri (Valenciennes, 1840) \\
\hline 162 & Thryssa dayi Wongaratna, 1983 & 204 & Arius maculatus (Thunberg, 1792) \\
\hline 163 & Thryssa dussumieri (Valenciennes, 1848) & 205 & Arius malabaricus Day, 1877 \\
\hline 164 & Thryssa malabarica (Bloch, 1795) & 206 & Arius platysomus Day, 1877 \\
\hline 165 & Thryssa mystax (Bloch \& Schneider, 1801) & 207 & Arius sagor (Hamilton, 1822) \\
\hline 166 & Thryssa purava (Hamilton, 1822) & 208 & Arius sona (Hamilton, 1822) \\
\hline 167 & Thryssa setirostris (Broussonet, 1782) & 209 & Arius tenuispinis Day, 1877 \\
\hline Family & Chirocentridae (wolf herrings) & 210 & Arius thalassinus (Rüppell, 1838) \\
\hline 168 & Chirocentrus dorab Forsskål, 1775 & 211 & Arius satparanus Chaudhuri 1916 \\
\hline 169 & Chirocentrus nudus Swainson, 1839 & 212 & Netuma serratus (Day, 1877) \\
\hline Family & Clupeidae (herrings, sardines) & 213 & Osteogeneiosus militaris (Linnaeus, 1758) \\
\hline 170 & Amblygaster clupeioides (Bleeker, 1849) & Family & Bagaridae (bagarid catfishes) \\
\hline 171 & Amblygaster leiogaster (Valenciennes, 1847) & 214 & Macarones armatus (Day, 1865) \\
\hline 172 & Anodontostoma chacunda (Hamilton, 1822) & 215 & Macarones gulio (Hamilton, 1822) \\
\hline 173 & Dussumieria acuta Valenciennes, 1847 & 216 & Macarones microphthalmus (Day, 1877) \\
\hline 174 & Dussumieria elopsoides Bleeker, 1849 & 217 & Mystus vittatus (Bloch, 1794) \\
\hline 175 & Escualosa thoracata (Valenciennes, 1847) & Sub division & EUTELEOSTEI \\
\hline 176 & Gudusia chapra (Hamilton, 1822) & Super order & STENOPTERYGII \\
\hline 177 & Nematalosa nasus (Bloch, 1795) & Order & STOMIIFORMES \\
\hline 178 & Opisthopterus tardoore (Cuvier, 1829) & Family & Stomiidae (viper fishes, dragon fishes) \\
\hline 179 & Pellona ditchela Valenciennes, 1847 & 218 & Chauliodus sloani Bloch \& Schneider, 1801 \\
\hline 180 & Pellona dayi Wongaratna, 1983 & 219 & Chauliodus pammelas (Alcock, 1892) \\
\hline 181 & Pellona dussumieri Valenciennes, 1847 & 220 & Stomias nebulosus Alcock, 1889 \\
\hline
\end{tabular}




\begin{tabular}{|c|c|}
\hline Family & Sternoptychidae (marine hatchetfishes) \\
\hline 221 & Argyropelecus alcocki (Goode \& Bean, 1896) \\
\hline 222 & Argyropelecus affinis Garman, 1899 \\
\hline 223 & Polyipnus indicus Schultz, 1961 \\
\hline 224 & Polyipnus asper Harold, 1994 \\
\hline 225 & Polyipnus omphus Baird, 1971 \\
\hline 226 & Polyipnus spinosus Günther, 1887 \\
\hline Super order & CYCLOSTOMATA \\
\hline Order & AULOPIFORMES \\
\hline Family & Synodontidae (lizardfishes) \\
\hline 227 & Saurida gracilis (Quoy \& Gaimard, 1824) \\
\hline 228 & Saurida longimanus Norman, 1939 \\
\hline 229 & Saurida tumbil (Bloch, 1795) \\
\hline 230 & Saurida undosquamis (Richardson, 1849) \\
\hline 231 & Synodus indicus (Day, 1873) \\
\hline 232 & Trachynocephalus myops (Bloch, 1792) \\
\hline Family & Chloropthalmidae (queen eyes) \\
\hline 233 & Chloropthalmus agassizi Bonnaparte , 1840 \\
\hline 234 & Chloropthalmus corniger Alcock, 1894 \\
\hline Super order & SCOPELOMORPHA \\
\hline Order & MYCTOPHIFORMES \\
\hline Family & Myctophidae (lanternfishes) \\
\hline 235 & Ceratoscopelus warmingii (Lutken, 1892) \\
\hline 236 & Diaphus effulgens (Goode \& Bean, 1896) \\
\hline 237 & Diaphus garmani Gilbert, 1906 \\
\hline 238 & Diaphus perspicillatus (Ogilby, 1898) \\
\hline 239 & Diaphus thiollierei Fowler, 1934 \\
\hline 240 & Myctophum fussunovi Becker \& Borodulina, 1971 \\
\hline 241 & Myctophum obtusirostre Taning, 1928 \\
\hline Super order & LAMPRIOMORPHA \\
\hline Order & LAMPRIFORMES \\
\hline Family & Regalecidae (oarfishes) \\
\hline 242 & Regaleus glesne Ascanius, 1772 \\
\hline Super order & POLYMIXIMORPHA \\
\hline Order & POLYMIXIFORMES \\
\hline Family & Polymixiidae (beardfishes) \\
\hline 243 & Polymixia berndti Gilbert, 1905 \\
\hline 244 & Polymixia busakhini Kotlyar, 1992 \\
\hline 245 & Polymixia fusca Kotthaus, 1970 \\
\hline 246 & Polymixia nobilis Lowe, 1836 \\
\hline Order & GADIFORMES \\
\hline Family & Macrouridae (grenadiers) \\
\hline 247 & Coelorinchus flabellispinnis (Alcock, 1894) \\
\hline 248 & Coelorinchus macrolepis Gilbert \& Hubbs, 1920 \\
\hline 249 & Macrurus fasciatus (Weber, 1913) \\
\hline 250 & Macrurus heterolepis (Alcock, 1889) \\
\hline 251 & Macrurus hispidus (Alcock, 1889) \\
\hline 252 & Macrurus investigatoris (Alcock, 1889) \\
\hline
\end{tabular}

\begin{tabular}{|c|c|}
\hline 253 & Macrurus brevirostris (Alcock, 1889) \\
\hline 254 & Nezumia semiquincunciata (Alcock, 1889) \\
\hline 255 & Nezumia propinqua (Gilbert \& Cramer, 1897) \\
\hline Order & BATRACHOIDIFORMES \\
\hline Famiy & Batrachoididae (toadfishes) \\
\hline 256 & Allenbatrachus grunniens (Linnaeus, 1758) \\
\hline 257 & Allenbatrachus reticulatus (Steindachner, 1870) \\
\hline 258 & Batrachomoeus trispinosus (Günther, 1861) \\
\hline 259 & Colletteichthys dussumieri (Valenciennes, 1837) \\
\hline 260 & Colletteichthys occidentalis Greenfield, 2012 \\
\hline 261 & Perulibatrachus aquilonarius Greenfield, 2005 \\
\hline Family & Bregmacerotidae (unicorn cod) \\
\hline 262 & Bregmaceros mcclellandi Thomson, 1840 \\
\hline Order & LOPHIIFORMES \\
\hline Family & Lophiidae (monkfishes) \\
\hline 263 & Lophiodes gracilimanus (Alcock, 1899) \\
\hline 264 & Lophiodes insidiator (Regan, 1921) \\
\hline 265 & Lophiodes lugubris (Alcock, 1894) \\
\hline 266 & Lophiodes setigerus (Vahl, 1797) \\
\hline Family & Antennariidae (frogfishes) \\
\hline 267 & Antennarius biocellatus (cuvier, 1817) \\
\hline 268 & Antennarius hispidus (Bloch \& Schneider, 1801) \\
\hline 269 & Antennarius indicus Schultz, 1964 \\
\hline 270 & Histrio histrio (Linnaeus, 1758) \\
\hline Family & Ogcocephalidae (batfishes) \\
\hline 271 & Coelophrys micropus (Alcock, 1891) \\
\hline 272 & Dibranchus nasutus Alcock, 1891 \\
\hline 273 & Dibranchus hystrix Garman, 1899 \\
\hline 274 & Halieutaea fumosa Alcock, 1894 \\
\hline 275 & Halieutaea coccinea Alcock, 1889 \\
\hline 276 & Halieutaea indica Annandale \& Jenkins, 1910 \\
\hline 277 & Halieutaea nigra Alcock, 1891 \\
\hline 278 & Halieutaea stellata (Vahl, 1797) \\
\hline Super order & ACANTHOPTERYGII \\
\hline Order & MUGILIFORMES \\
\hline Family & Mugilidae (mullets) \\
\hline 279 & Chelon tade (Forsskål, 1775)1 \\
\hline 280 & Liza Kluzingeri (Day, 1888) \\
\hline 281 & Liza melinopterus (Valenciennes, 1836) \\
\hline 282 & Liza parsia (Hamilton, 1822) \\
\hline 283 & Ellochelon vaigiensis (Quoy \& Gaimard, 1825) ${ }^{2}$ \\
\hline 284 & Liza carinata (Valenciennes, 1836) \\
\hline 285 & Liza dussumeiri (Valenciennes, 1836) \\
\hline 286 & Liza macrolepis (Smith, 1849) \\
\hline 287 & Liza mandapamensis (Thomson, 1997) \\
\hline 288 & Liza subviridis (Valenciennes, 1836) \\
\hline 289 & Moolgarda cunnesius (Valenciennes, 1836) \\
\hline 290 & Mugil cephalus Linnaeus, 1758 \\
\hline
\end{tabular}

Synonym: Liza planiceps (Valenciennes, 1836)

2Synonym: Liza vaigiensis (Quoy \& Gaimard, 1825)

3Synonym: Valamugil cunnesius (Valenciennes, 1836) 
Check list of fishes of Gulf of Mannar, India

\begin{tabular}{|c|c|}
\hline 291 & Planiliza alata ( Steindachner, 1892) \\
\hline 292 & Rhinomugil corsula (Hamilton, 1822) \\
\hline 293 & Valamugil buchanani (Bleeker, 1853) \\
\hline 294 & Valamugil seheli (Bleeker, 1858) \\
\hline 295 & Valamugil speigleri (Bleeker, 1858) \\
\hline Super order & ATHERINEA \\
\hline Order & ATHERINIFORMES \\
\hline Family & Atherinidae (silversides) \\
\hline 296 & Atherina lacunosa Forster, 1801, \\
\hline 297 & Atherina melanostigma Day, 1876 \\
\hline 298 & Atherina forskalii Rüppell, 1838 \\
\hline 299 & Atherinomorus duodecimalis (Valenciennes, 1835) \\
\hline Super order & CYPRINODONTEA \\
\hline Order & BELONIFORMES \\
\hline Family & Exocoetidae (flyingfishes) \\
\hline 300 & Cheilopogon spilopterus (Valenciennes 1847) \\
\hline 301 & Cheilopogon furcatus (Mitchill, 1815) \\
\hline 302 & Cypselurus comatus (Mitchill, 1815) \\
\hline 303 & Cypselurus oligolepis (Bleeker, 1865) \\
\hline 304 & Exocoetus monocirrhus Richardson, 1846 \\
\hline 305 & Exocoetus volitans Linnaeus 1758 \\
\hline 306 & Hirundichthys oxycephalus (Bleeker, 1853) \\
\hline 307 & Hirundichthys coromandelensis (Hornell, 1923) \\
\hline 308 & Paraexocoetus branchypterus (Richardson, 1846) \\
\hline Family & Hemirhamphidae (halfbeaks) \\
\hline 309 & Hemirhamphus far (Forsskål, 1775) \\
\hline 310 & Hemirhamphus lutkei Valenciennes, 1847 \\
\hline 311 & Hemirhamphus robustus Günther, 1866 \\
\hline 312 & Hemirhamphus marginatus (Forsskål, 1775) \\
\hline 313 & Hemirhamphus quoyi (Valenciennes, 1847) \\
\hline 314 & Hemirhamphus gaimardi (Valenciennes, 1847) \\
\hline 315 & Hyporhamphus dussumieri (Valenciennes, 1847) \\
\hline 316 & Hyporhamphus xanthopterus (Valenciennes, 1847) \\
\hline 317 & Rhyncorhamphus malabaricus Collette, 1976 \\
\hline Family & Belonidae (fullbeaks) \\
\hline 318 & Ablennes hians (Valenciennes, 1846) \\
\hline 319 & Platybelone arga/us (Lesueur, 1821) \\
\hline 320 & Strongy/ura leiura (Bleeker, 1850) \\
\hline 321 & Strongylura scapularis (Jordan \&Gilbert, 1882) \\
\hline 322 & Strongylura strongy/ura (van Hasselt, 1823) \\
\hline 323 & Tylosurus choram (Rüppell, 1837) \\
\hline 324 & Tylosurus crocodilus (Peron \& Lesueur, 1821) \\
\hline 325 & Tylosurus melanotus (Bleeker, 1850) \\
\hline Order & CYPRINODONTIFORMES \\
\hline Family & Aplocheilidae (rivulines) \\
\hline 326 & Aplocheilus panchax (Hamilton, 1822) \\
\hline Order & BERCYFORMES \\
\hline Family & Berycidae (squirrelfishes) \\
\hline
\end{tabular}

\begin{tabular}{|c|c|}
\hline 327 & Beryx delphini Valenciennes, 1833 \\
\hline 328 & Centroberyx gerrardi (Günther, 1887) \\
\hline 329 & Centroberyx rubricaudus Liu \& Shen, 1985 \\
\hline Family & Trachichthyidae (slimeheads) \\
\hline 330 & Hoplostethus mediterraneus ( Cuvier, 1829) \\
\hline Family & Holocentridae (squirrelfishes \& soldierfishes) \\
\hline 331 & Holocentrum lacteoguttatum Cuvier \& Valenciennes, 1829 \\
\hline 332 & Myripristis adusta Bleeker, 1853 \\
\hline 333 & Myripristis murdjan (Forsskål, 1775,) \\
\hline 334 & Myripristis berndti (Jordan \& Evermann, 1903) \\
\hline 335 & Neoniphon sammara (Forsskål, 1775) \\
\hline 336 & Sargocentron caudimaculatum (Rüppell, 1838) \\
\hline 337 & Sargocentron diadema (Lacepède, 1802) \\
\hline 338 & Sargocentron rubrum (Forsskål, 1775) \\
\hline 339 & Sargocentron spiniferum (Forsskål, 1775) \\
\hline Order & ZEIFORMES \\
\hline Family & Zeidae (dories) \\
\hline 340 & Zenopsis conchifer (Lowe, 1852) \\
\hline Order & GASTEROSTEIFORMES \\
\hline Family & Pegasidae (seamoths) \\
\hline 341 & Pegasus laternarius Cuvier, 1816 \\
\hline 342 & Pegasus volitans Linnaeus, 1758 \\
\hline 343 & Parapegasus natans Linnaeus, 1758 \\
\hline 344 & Eurypegasus draconius (Linnaeus, 1766) \\
\hline Order & SYNGNATHIFORMES \\
\hline Family & Syngnathidae (pipefishes \& seahorses) \\
\hline 345 & Hippocampus fuscus Rüppell, 1838 \\
\hline 346 & Hippocampus histrix Kaup, 1856 \\
\hline 347 & Hippocampus kelloggi Jordan \& Snyder, 1901 \\
\hline 348 & Hippocampus kuda Bleeker, 1852 \\
\hline 349 & Hippocampus spinosissimus Weber, 1913 \\
\hline 350 & Hippocampus trimaculatus Leach, 1814 \\
\hline 351 & Micrognathus brevirostris (Rüppell, 1838) \\
\hline 352 & Syngnathoides biaculeatus (Bloch, 1785) \\
\hline 353 & Trachyrhamphus longirostris Kaup, 1856 \\
\hline 354 & Trachyrhamphus serratus (Temminck \& Schlegel, 1850) \\
\hline Family & Fistularidae (cornetfishes) \\
\hline 355 & Fistularia petimba Lacepède, 1803 \\
\hline 356 & Fistularia paradoxus (Pallas, 1770) \\
\hline 357 & Fistularia villosa Klunzinger, 1871 \\
\hline Family & Centriscidae (shrimpfishes, snipefishes) \\
\hline 358 & Aeoliscus punctulatus (Bianconi, 1854) \\
\hline 359 & Aeoliscus strigatus (Günther, 1861) \\
\hline 360 & Centriscus scutatus Linnaeus, 1758 \\
\hline 361 & Centriscus humerosus Richardson, 1846 \\
\hline Order & SCORPAENIFORMES \\
\hline Family & Scorpaenidae (scorpionfishes) \\
\hline 362 & Dendrochirus zebra (Cuvier, 1829) \\
\hline 363 & Minous inermis Alcock, 1889 \\
\hline 364 & Minous monodacty/us (Bloch \& Schneider, 1801) \\
\hline
\end{tabular}




\begin{tabular}{|c|c|c|c|}
\hline 365 & Parapterois heterurus (Bleeker, 1856) & 407 & Ambassis nalua (Hamilton, 1822) \\
\hline 366 & Pterois antennata (Bloch, 1787) & 408 & Parambassis dayi (Bleeker, 1874) \\
\hline 367 & Pterois russelii Bennett, 1831 & Family & Latidae (lates perches) \\
\hline 368 & Pterois volitans (Linnaeus, 1758) & 409 & Psammoperca waigiensis (Cuvier, 1828) \\
\hline 369 & Rhinopias eschmeyeri Conde, 1977 & 410 & Lates calcarifer (Bloch, 1790) \\
\hline 370 & Scorpaenodes corallinus Smith, 1957 & Family & Serranidae (sea bassess, groupers) \\
\hline 371 & Scorpaenodes parvipinnis (Garrett, 1864) & 411 & Aulacocephalus temminckii Bleeker, 1854 \\
\hline 372 & Scorpaenopsis gibbosa (Bloch \& Schneider, 1801) & 412 & Cephalopholis aurantia (Valenciennes, 1828) \\
\hline Family & Tetrarogidae & 413 & Cephalopholis argus Schneider, 1801 \\
\hline 373 & Ablabys binotatus (Peters, 1855) & 414 & Cephalopholis boenak (Bloch, 1790) \\
\hline Family & Platycephalidae (flatheads) & 415 & Cephalopholis formosa (Shaw, 1804) \\
\hline 374 & Cociella crocodila (Cuvier, 1829) & 416 & Cephalopholis leopardus (Lacepède, 1801) \\
\hline 375 & Cociella punctata (Cuvier, 1829) & 417 & Cephalopholis polleni (Bleeker, 1868) \\
\hline 376 & Grammoplites scaber (Linnaeus, 1758) & 418 & Cephalopholis sonnerati (Valenciennes, 1828) \\
\hline 377 & Grammoplites suppositus (Troschel, 1840) & 419 & Epinephelus fasciatus (Forsskål, 1775) \\
\hline 378 & Inegocia japonica (Cuvier, 1829) & 420 & Epinephelus polyphekadion (Bleeker, 1849) \\
\hline 379 & Kumococius rodericensis (Cuvier, 1829) & 421 & Epinephelus areolatus (Forsskål, 1775) \\
\hline 380 & Sunagocia carbunculus (Valenciennes, 1833) & 422 & Epinephelus bleekeri (Vaillant, 1878) \\
\hline 381 & Platycephalus indicus (Linnaeus, 1758) & 423 & Epinephelus coeruleopunctatus (Bloch, 1790) \\
\hline 382 & Platycephalus macracanthus (Bleeker, 1869) & 424 & Epinephelus chlorostigma (Valenciennes, 1828) \\
\hline 383 & Rogadius asper (Cuvier, 1829) & 425 & Epinephelus diacanthus (Valenciennes, 1828) \\
\hline 384 & Rogadius serratus (Cuvier, 1829) & 426 & Epinephelus flavocaeruleus (Lacepède, 1802) \\
\hline 385 & Rogadius tuberculata (Cuvier, 1829) & 427 & Epinephe/us fuscoguttatus (Forsskål, 1775) \\
\hline 386 & Rogadius welanderi (Schultz, 1966) & 428 & Epinephelus hexagonatus (Forster, 1801) \\
\hline 387 & Sunagocia carbunculus (Valenciennes, 1833) & 429 & Epinephelus longispinis (Kner, 1864) \\
\hline 388 & Sunagocia otaitensis (Cuvier, 1829) & 430 & Epinephelus malabaricus (Bloch \& Schneider, 1801) \\
\hline \multirow[t]{2}{*}{ Family } & \multirow{2}{*}{$\begin{array}{l}\text { Peristediidae (armored searobins or armored } \\
\text { gurnards) }\end{array}$} & 431 & Epinephelus merra Bloch,1793 \\
\hline & & 432 & Epinephe/us miniatus (Forsskål, 1775) \\
\hline 389 & Peristedion adeni (Loyd, 1907) & 433 & Epinephelus morrhua (Valenciennes, 1833) \\
\hline 390 & Peristedion miniatum Goode, 1880 & 434 & Epinephelus sexfasciatus (Valenciennes, 1828) \\
\hline Order & POLYNEMIFORMES & 435 & Epinephelus tauvina (Forsskål, 1775) \\
\hline Family & Polynemidae (threadfins) & 436 & Epinephelus undulosus (Quoy \& Gaimard, 1824) \\
\hline 391 & Eleutheronema tetradacty/um (Shaw, 1804) & 437 & Grammistes sexlineatus (Thunberg, 1792) \\
\hline 392 & Filimanus heptadactyla (Cuvier, 1829) & 438 & Pogonoperca punctata (Valenciennes, 1830) \\
\hline 393 & Filimanus similis Feltes, 1991 & 439 & Promicrops lanceolatus (Bloch, 1790) \\
\hline 394 & Polydactylus indicus (Shaw, 1804) & 440 & Variola louti (Forsskål, 1775) \\
\hline 395 & Polydacty/us sextarius (Bloch \& Schneider, 1801) & Family & Priacanthidae (bigeyes or catalufas) \\
\hline 396 & Polynemus dubius Bleeker, 1853 & 441 & Priacanthus hamrur Forsskål, 1775 \\
\hline 397 & Polynemus indicus (Shaw, 1804) & 442 & Priacanthus cruentatus (Lacepède, 1801) \\
\hline 398 & Polynemus multifilis (Temminck \& Schlegel, 1843) & 443 & Priacanthus tayenus Richardson, 1846 \\
\hline 399 & Polynemus multiradiatus (Günther, 1860) & 444 & Priacanthus blochii Bleeker, 1853 \\
\hline 400 & Polynemus paradiseus Linnaeus, 1758 & 445 & Priacanthus macracanthus Cuvier, 1829 \\
\hline 401 & Polynemus plebius (Broussonet, 1782) & Family & Sillaginidae (smelt-whitings) \\
\hline 402 & Polynemus sexfilis (Valenciennes, 1831) & 446 & Sillago sihama (Forsskål, 1775) \\
\hline 403 & Polynemus sextarius Bloch \& Schneider, 1801 & 447 & Sillago maculata Quoy \& Gaimard, 1824 \\
\hline Order & PERCIFORMES & 448 & Sillago aeolus Jordan \& Evermann, 1902 \\
\hline Family & Ambassidae (asiatic glassfishes) & 449 & Sillago vittata McKay, 1985 \\
\hline 404 & Ambassis commersonii Cuvier, 1828 & 450 & Sillago intermedius Wongratana, 1977 \\
\hline 405 & Ambassis gymnocephalus (Lacepède, 1802) & 451 & Sillago lutea McKay, 1985 \\
\hline 406 & Ambassis malabaricus Valenciennes, 1849 & 452 & Sillago vincenti McKay, 1980 \\
\hline
\end{tabular}




\begin{tabular}{|c|c|}
\hline Family & Malacanthidae (tilefishes) \\
\hline 453 & Malacanthus latovittatus (Lacepède, 1801) \\
\hline Family & Lactaridae (false trevally) \\
\hline 454 & Lactarius lactarius (Bloch \& Schneider, 1801) \\
\hline Family & Coryphaenidae (dolphinfishes) \\
\hline 455 & Coryphaena hippurus Linnaeus, 1758 \\
\hline 456 & Coryphaena equiselis Linnaeus, 1758 \\
\hline Family & Rachycentridae (cobia) \\
\hline 457 & Rachycentron canadum (Linnaeus, 1766) \\
\hline Family & Echeneidae (remoras, shark suckers) \\
\hline 458 & Echeneis naucrates Linnaeus, 1758 \\
\hline 459 & Remora remora (Linnaeus, 1758) \\
\hline 460 & Remora osteochir (Cuvier, 1829) \\
\hline Family & Apogonidae (cardinalfishes) \\
\hline 461 & Apogon aureus (Lacepède, 1802) \\
\hline 462 & Apogon compressus (Smith \& Radcliffe, 1911) \\
\hline 463 & Apogon ellioti Day, 1875 \\
\hline 464 & Apogon endekataenia Bleeker, 1852 \\
\hline 465 & Apogon enneastigma Rüppell, 1838 \\
\hline 466 & Apogon fraenatus (Valenciennes, 1832) \\
\hline 467 & Apogon kallopterus Bleeker, 1856 \\
\hline 468 & Apogon leptacanthus Bleeker, 1856 \\
\hline 469 & Apogon lineatus Temminck \& Schlegel, 1843 \\
\hline 470 & Apogon nigricans Day, 1875 \\
\hline 471 & Apogon nigripinnis Cuvier, 1828 \\
\hline 472 & Apogon nigrofasciatus Lachner, 1953 \\
\hline 473 & Apogon novemfasciatus Cuvier, 1828 \\
\hline 474 & Apogon quadrifasiatus Cuvier, 1828 \\
\hline 475 & Apogon quinquestriatus Regan, 1908 \\
\hline 476 & Apogon savayensis Günther, 1872 \\
\hline 477 & Apogon septemstriatus Günther, 1880 \\
\hline 478 & Apogon smithi (Kotthaus, 1970) \\
\hline 479 & Apogon truncatus Bleeker, 1855 \\
\hline 480 & Apogonichthyoides erdmanni Fraser \& Allen, 2011 \\
\hline 481 & Apogonichthyoides umbratilis Fraser \& Allen, 2010 \\
\hline 482 & Apogonichthys ocellatus (Weber, 1913) \\
\hline 483 & Apogonichthyoides pseudotaeniatus (Gon, 1986) \\
\hline 484 & Archamia macroptera (Cuvier, 1828) \\
\hline 485 & Cheilodipterus artus Smith, 1961 \\
\hline 486 & Cheilodipterus macrodon (Lacepède, 1802) \\
\hline 487 & Cheilodipterus quinquelineatus Cuvier, 1828 \\
\hline 488 & Foa brachygramma (Jenkins, 1903) \\
\hline 489 & Fowleria marmorata (Alleyne \& Macleay, 1877) \\
\hline 490 & Glossamia arguni Hadiaty \& Allen, 2011 \\
\hline 491 & Glossamia sandei (Weber, 1907) \\
\hline 492 & Glossamia trifasciata (Weber, 1913) \\
\hline 493 & Neamia octospina Smith \& Radcliffe, 1912 \\
\hline 494 & Ostorhinchus apogonoides (Bleeker, 1856) \\
\hline 495 & Ostorhinchus chrysotenia ( Bleeker, 1851) \\
\hline 496 & Ostorhinchus cyanosoma ( Bleeker, 1853) \\
\hline
\end{tabular}

\begin{tabular}{|c|c|}
\hline 497 & Ostorhinchus dispar (Fraser \& Randall, 1976) \\
\hline 498 & Ostorhinchus fleurieu Lacepède, 1802 \\
\hline 499 & Ostorhinchus hoevenii ( Bleeker, 1854) \\
\hline 500 & Ostorhinchus lateralis (Valenciennes, 1832) \\
\hline 501 & Pristiapogon fraenatus (Valenciennes, 1832) \\
\hline 502 & Synagrops adeni Kotthaus, 1970 \\
\hline 503 & Synagrops japonicus (Döderlein, 1883) \\
\hline Family & Carangidae (jacks \& pompanos) \\
\hline 504 & Alectis ciliaris (Bloch, 1787) \\
\hline 505 & Alectis indica (Rüppell, 1830) \\
\hline 506 & Alepes djedaba (Forsskål, 1775) \\
\hline 507 & Alepes kleinii (Bloch, 1793) \\
\hline 508 & Alepes melanoptera (Swainson, 1839) \\
\hline 509 & Alepes vari (Cuvier, 1833) \\
\hline 510 & Atropus atropos (Bloch \& Schneider, 1801) \\
\hline 511 & Atule mate (Cuvier, 1833) \\
\hline 512 & Carangoides armatus (Rüppell, 1830) \\
\hline 513 & Carangoides bajad (Forsskål, 1775) \\
\hline 514 & Carangoides coeruleopinnatus (Rüppell, 1830) \\
\hline 515 & Carangoides chrysophrys (Cuvier, 1833) \\
\hline 516 & Carangoides dinema Bleeker, 1851 \\
\hline 517 & Carangoides equula (Temminck \& Schlegel, 1844) \\
\hline 518 & Carangoides ferdau (Forsskål, 1775) \\
\hline 519 & Carangoides fulvoguttatus (Forsskål, 1775) \\
\hline 520 & Carangoides gymnostethus (Cuvier 1833) \\
\hline 521 & Carangoides hedlandensis (Whitley, 1934 \\
\hline 522 & Carangoides malabaricus (Bloch \& Schneider, 1801) \\
\hline 523 & Carangoides oblongus (Cuvier, 1833) \\
\hline 524 & Carangoides orthogrammus (Jordan \& Gilbert, 1881) \\
\hline 525 & Carangoides plagiotaenia Bleeker, 1857 \\
\hline 526 & Carangoides praeustus Bennett, 1830 \\
\hline 527 & Carangoides talamparoides Bleeker, 1852 \\
\hline 528 & Carangoides uii (Wakiya, 1924) \\
\hline 529 & Caranx carangus (Bloch, 1793) \\
\hline 530 & Caranx ignobilis (Forsskål, 1775) \\
\hline 531 & Caranx lugubris Poey, 1860 \\
\hline 532 & Caranx melampygus Cuvier, 1833 \\
\hline 533 & Caranx papuensis Alleyne \&Macleay, 1877 \\
\hline 534 & Caranx sem Cuvier, 1833 \\
\hline 535 & Caranx sexfasciatus Quoy \& Gaimard, 1825 \\
\hline 536 & Caranx tille Cuvier, 1833 \\
\hline 537 & Decapterus kurroides Bleeker, 1855 \\
\hline 538 & Decapterus macarellus Cuvier, 1833 \\
\hline 539 & Decapterus macrosoma Bleeker, 1851 \\
\hline 540 & Decapterus russelli (Rüppell, 1830) \\
\hline 541 & Decapterus tab/Berry, 1968 \\
\hline 542 & Elagatis bipinnulata (Quoy \& Gaimard, 1825) \\
\hline 543 & Gnathanodon speciosus (Forsskål, 1775) \\
\hline 544 & Megalaspis cordyla (Linnaeus, 1758) \\
\hline 545 & Naucrates ductor (Linnaeus, 1758) \\
\hline
\end{tabular}




\begin{tabular}{|c|c|c|c|}
\hline 546 & Parastromateus niger (Bloch, 1795) & 589 & Aphareus furca (Lacepède, 1801) \\
\hline 547 & Scomberoides commersonnianus Lacepède, 1801 & 590 & Aprion virescens Valenciennes, 1830 \\
\hline 548 & Scomberoides lysan (Forsskål, 1775) & 591 & Aphareus rutilans Cuvier, 1830 \\
\hline 549 & Scomberoides tala (Cuvier, 1832) & 592 & Etelis coruscans Valenciennes, 1862 \\
\hline 550 & Scomberoides tol (Cuvier, 1832) & 593 & Etelis carbunculus Cuvier, 1828 \\
\hline 551 & Selar boops (Cuvier, 1833) & 594 & Lipocheilius carnolabrum (Chan, 1970) \\
\hline 552 & Selar crumenophthalmus (Bloch, 1793) & 595 & Lutjanus argentimaculatus (Forsskål, 1775) \\
\hline 553 & Selaroides leptolepis (Cuvier, 1833) & 596 & Lutjanus bengalensis (Bloch, 1790) \\
\hline 554 & Seriola rivoliana Valenciennes, 1833 & 597 & Lutjanus biguttatus (Valenciennes, 1830) \\
\hline 555 & Seriola dumerili (Risso, 1810) & 598 & Lutjanus bohar (Forsskål, 1775) \\
\hline 556 & Seriolina nigrofasciata (Rüppell, 1829) & 599 & Lutjanus boutton (Lacepède, 1802) \\
\hline 557 & Trachinotus baillonii (Lacepède, 1801) & 600 & Lutjanus coeruleolineatus (Rüppell, 1838) \\
\hline 558 & Trachinotus blochii (Lacepède, 1801) & 601 & Lutjanus decussatus (Cuvier, 1828) \\
\hline 559 & Trachinotus coppingeri Günther, 1884 & 602 & Lutjanus ehrenbergii (Peter, 1869) \\
\hline 560 & Trachinotus mookalee Cuvier, 1832 & 603 & Lutjanus erythropterus Bloch, 1790 \\
\hline 561 & Trachinotus russelii Cuvier, 1832 & 604 & Lutjanus fulviflamma (Forsskål, 1775) \\
\hline 562 & Ulua mentalis (Cuvier, 1833) & 605 & Lutjanus fulvus (Schneider, 1801) \\
\hline 563 & Uraspis helvola (Forster, 1801) & 606 & Lutjanus gibbus (Forster, 1801) \\
\hline 564 & Uraspis secunda (Poey, 1860) & 607 & Lutjanus guilcheri Fourmanoir, 1959 \\
\hline 565 & Uraspis uraspis (Günther, 1860) & 608 & Lutjanus johnii (Bloch, 1792) \\
\hline Family & Menidae (moonfishes) & 609 & Lutjanus kasmira (Forsskål, 1775) \\
\hline 566 & Mene maculata (Bloch \& Schneider, 1801) & 610 & Lutjanus lemniscatus (Valenciennes, 1828) \\
\hline Family & Leiognathidae (silverbellies, ponyfishes) & 611 & Lutjanus lineolatus (Rüppell, 1829) \\
\hline 567 & Aurigequula longispina (Valenciennes, 1835)' & 612 & Lutjanus lunulatus (Park, 1797) \\
\hline 568 & Aurigequula fasciata (Lacepède, 1803)² & 613 & Lutjanus lutjanus Bloch, 1790 \\
\hline 569 & Equulites absonditus Chakrabarty \& Sparks, 2010 & 614 & Lutjanus madras (Valenciennes, 1831) \\
\hline 570 & Equulites elongatus (Günther, 1874)3 & 615 & Lutjanus malabaricus (Bloch \& Schneider, 1801) \\
\hline 571 & Equulites leuciscus (Günther, 1860) ${ }^{4}$ & 616 & Lutjanus monostigma (Cuvier, 1828) \\
\hline 572 & Eubleekeria rapsoni (Munro, 1964) & 617 & Lutjanus quinquelineatus (Bloch, 1790) \\
\hline 573 & Eubleekeria splendens (Cuvier, 1829)5 & 618 & Lutjanus rivulatus (Cuvier, 1828) \\
\hline 574 & Gazza achlamys Jordan \& Starks, 1917 & 619 & Lutjanus rufolineatus (Valenciennes, 1830) \\
\hline 575 & Gazza minuta (Bloch, 1795) & 620 & Lutjanus indicus Allen, White \& Erdmann, 2013 \\
\hline 576 & Gazza shettyi Jayabalan, 1986 & 621 & Lutjanus sebae (Cuvier, 1816) \\
\hline 577 & Karalla daura (Cuvier, 1829)6 & 622 & Lutjanus sanguineus (Cuvier, 1828) \\
\hline 578 & Karalla dussumieri (Valenciennes, 1835)7 & 623 & Lutjanus vitta (Quoy \& Gairmard, 1824) \\
\hline 579 & Leiognathus smithrusti Ramsay \& Ogilby 1886 & 624 & Pinjalo pinjalo (Bleeker, 1850) \\
\hline 580 & Leiognathus jonesi James, 1971 & 625 & Pinjalo lewisi (Randall, Allen \& Anderson, 1987) \\
\hline 581 & Leiognathus berbis (Valenciennes, 1835) & 626 & Pristipomoides filamentosus (Valenciennes, 1830) \\
\hline 582 & Leiognathus equulus (Forsskål, 1775) & 627 & Pristipomoides auricilla (Jordan, Evermann \& Tanaka, 1927) \\
\hline 583 & Nuchequula nuchalis (Temminck \& Schlegel, 1845) & 628 & Pristipomoides multidens (Day, 1871) \\
\hline 584 & Nuchequula mannusella Chakrabarty \& Sparks, 2007 & 629 & Pristipomoides typus Bleeker, 1852 \\
\hline 585 & Photopectoralis bindus (Valenciennes, 1835)8 & 630 & Pristipomoides zonatus (Valenciennes, 1830) \\
\hline 586 & Secutor insidiator (Bloch, 1787) & 631 & Macolor niger (Forsskål, 1775) \\
\hline 587 & Secutor ruconius (Hamilton, 1822) & 632 & Paracaesio sordida Abe \& Shinohara, 1962. \\
\hline Family & Bramidae (breams) & Family & Lobotidae (triplettails) \\
\hline 588 & Brama rajii (Bloch \&Schneider, 1801) & 633 & Lobotes surinamensis (Bloch, 1790) \\
\hline Family & Lutjanidae (snappers) & Family & Caesionidae (fusiliers) \\
\hline
\end{tabular}

Synonym: Leiognathus longispinis (Valenciennes, 1835)

2 Synonym: Leiognathus fasciatus (Lacepede, 1803)

${ }^{3}$ Synonym: Leiognathus elongatus (Gunther, 1874)

${ }^{4}$ Synonym: Leiognathus leuciscus (Gunther, 1860)
5 Synonym: Leiognathus splendens (Cuvier, 1829)

6 Synonym: Leiognathus daura (Cuvier, 1829)

7 Synonym: Leiognathus dussumieri (Valenciennes, 1835)

8 Synonym: Leiognathus bindus (Valenciennes, 1835) 
Check list of fishes of Gulf of Mannar, India

\begin{tabular}{|c|c|}
\hline 634 & Caesio cuning (Bloch, 1791) \\
\hline 635 & Caesio chrysozona Cuvier, 1830 \\
\hline 636 & Caesio erythrogaster Cuvier, 1830 \\
\hline 637 & Caesio caerulaurea Lacepède, 1801 \\
\hline 638 & Pterocaesio tile (Cuvier, 1830) \\
\hline Family & Gerreidae (mojarras) \\
\hline 639 & Gerres argyreus (Forster, 1801) \\
\hline 640 & Gerres abbreviatus Bleeker, 1850 \\
\hline 641 & Gerres filamentous Cuvier, 1829 \\
\hline 642 & Gerres limbatus (Cuvier, 1830) \\
\hline 643 & Gerres oblongus Cuvier, 1830 \\
\hline 644 & Gerres oyena (Forsskål, 1775) \\
\hline 645 & Pentaprion longimanus (Cantor, 1849) \\
\hline Family & Haemulidae (sweetlips, grunts) \\
\hline 646 & Diagramma pictum (Thunberg, 1792) \\
\hline 647 & Plectorhinchus gibbosus (Lacepède, 1802) \\
\hline 648 & Plectorhinchus flavomaculatus (Cuvier, 1830) \\
\hline 649 & Plectorhinchus nigrus (Cuvier, 1830) \\
\hline 650 & Plectorhinchus schotaf (Forsskål, 1775) \\
\hline 651 & Plectorhinchus vittatus (Linnaeus, 1758) \\
\hline 652 & Plectorhinchus gibbosus (Lacepède, 1802) \\
\hline 653 & Plectorhinchus lineatus (Linnaeus, 1758) \\
\hline 654 & Plectorhinchus orientalis (Bloch, 1793) \\
\hline 655 & Pomadasys argyreus (Valenciennes, 1833) \\
\hline 656 & Pomadasys commersonii (Lacepède, 1801) \\
\hline 657 & Pomadasys auritus (Cuvier, 1830) \\
\hline 658 & Pomadasys trifasciatus (Fowler, 1937) \\
\hline 659 & Pomadasys hasta (Bloch, 1790) \\
\hline 660 & Pomadasys maculatum (Bloch, 1793) \\
\hline 661 & Pomadasys olivaceus (Day, 1875) \\
\hline 662 & Pomadasys argenteus (Forsskål, 1775) \\
\hline 663 & Pomadasys kaakan (Cuvier, 1830) \\
\hline Family & Nemipteridae (Threadfin breams) \\
\hline 664 & Nemipterus nematophorus (Bleeker, 1854) \\
\hline 665 & Nemipterus peronii (Valenciennes, 1830) \\
\hline 666 & Nemipterus bipunctatus (Valenciennes, 1830) \\
\hline 667 & Nemipterus delagoae Smith, 1941 \\
\hline 668 & Nemipterus japonicus (Bloch, 1791) \\
\hline 669 & Nemipterus mesoprion (Bleeker, 1853) \\
\hline 670 & Nemipterus zysron (Bleeker, 1856) \\
\hline 671 & Parascolopsis eriomna (Jordan \& Richardson, 1909) \\
\hline 672 & Parascolopsis inermis (Temminck \& Schlegel,1843) \\
\hline 673 & Scolopsis bimaculata Rüppell, 1828 \\
\hline 674 & Scolopsis vosmeri (Bloch, 1792) \\
\hline Family & Lethrinidae (pigface breams, emperors) \\
\hline 675 & Gymnocranius griseus (Temminck \& Schlegel,1844) \\
\hline 676 & Lethrinus lentjan (Lacepède, 1802) \\
\hline 677 & Lethrinus mahsena (Forsskål, 1775) \\
\hline 678 & Lethrinella miniatus (Forster, 1801) \\
\hline 679 & Lethrinus fletus Whitley, 1943 \\
\hline
\end{tabular}

\begin{tabular}{|c|c|}
\hline 680 & Lethrinus harak (Forsskål, 1775) \\
\hline 681 & Lethrinus microdon Valenciennes, 1830 \\
\hline 682 & Lethrinus nebulosus (Forsskål, 1775) \\
\hline 683 & Lethrinus ornatus Valenciennes, 1830 \\
\hline 684 & Lethrinus ramark Forsskål, 1775 \\
\hline 685 & Lethrinus reticulatus Valenciennes, 1830 \\
\hline 686 & Lethrinus rhodopterus Bleeker, 1852 \\
\hline 687 & Lethrinus cineraus Valenciennes ,1830 \\
\hline 688 & Lethrinus variegatus Valenciennes, 1846 \\
\hline 689 & Monotaxis grandoculis (Forsskål, 1775) \\
\hline 690 & Plectorhinchus nigrus (Cuvier, 1830) \\
\hline Family & Sparidae (porgies, seabreams) \\
\hline 691 & Acanthopagrus berda (Forsskål, 1775) \\
\hline 692 & Acanthopagrus latus (Houttuyn, 1782) \\
\hline 693 & Argyrops filamentosus (Valenciennes, 1830) \\
\hline 694 & Argyrops spinifer (Forsskål, 1775) \\
\hline 695 & Crenidens crenidens (Forsskål, 1775) \\
\hline 696 & Rhabdosargus sarba (Forsskål, 1775) \\
\hline 697 & Sparidentex hasta (Valenciennes, 1830) \\
\hline Family & Sciaenidae (drums or croakers) \\
\hline 698 & Atrobucca nibe (Jordan \& Thompson, 1911) \\
\hline 699 & Johnieops coitor (Hamilton, 1822) \\
\hline 700 & Johnius sina (Cuvier, 1830) \\
\hline 701 & Johnius vogleri (Bleeker, 1853) \\
\hline 702 & Johnius glaucus (Day, 1876) \\
\hline 703 & Johnius amblycephalus (Bleeker, 1855) \\
\hline 704 & Johnius aneus (Bloch, 1793) \\
\hline 705 & Johnius carouna (Cuvier, 1830) \\
\hline 706 & Johnius carutta Bloch, 1793 \\
\hline 707 & Johnius dussumieri (Cuvier, 1830) \\
\hline 708 & Johnius elongatus Lal Mohan, 1976 \\
\hline 709 & Johnius macrolepidotus (Bloch \& Schneider, 1801) \\
\hline 710 & Johnius macrorhynus (Lal Mohan, 1976) \\
\hline 711 & Johnius mannarensis Lal Mohan, 1971 \\
\hline 712 & Johnius maropterus (Bleeker, 1853) \\
\hline 713 & Kathala axillaris (Cuvier, 1830) \\
\hline 714 & Nibea albida (Cuvier, 1830) \\
\hline 715 & Nibea diacanthus (Lacepède, 1802) \\
\hline 716 & Nibea maculata (Bloch \& Schneider, 1801) \\
\hline 717 & Nibea soldado (Lacepède, 1802) \\
\hline 718 & Otolithes cuvieri Trewavas, 1974 \\
\hline 719 & Otolithes ruber (Bloch \& Schneider, 1801) \\
\hline 720 & Otolithes argenteus Cuvier, 1830 \\
\hline 721 & Otolithes maculatus Cuvier, 1830 \\
\hline 722 & Otolithoides pama (Hamilton, 1822) \\
\hline 723 & Otolithoides biauritus (Cantor, 1849) \\
\hline 724 & Panna heterolepis Trewavas, 1977 \\
\hline 725 & Panna microdon (Bleeker, 1849) \\
\hline 726 & Pennahia anea (Bloch, 1793) \\
\hline 727 & Pennahia macrophthalmus (Bleeker, 1850) \\
\hline
\end{tabular}




\begin{tabular}{|c|c|c|c|}
\hline 728 & Protonibea diacanthus (Lacepède, 1802) & 771 & Chaetodon lineolatus (Cuvier, 1831) \\
\hline 729 & Pseudoscianea indica Tang, 1937 & 772 & Chaetodon lunula (Lacepède, 1802) \\
\hline 730 & Pseudoscianea coibor (Hamilton, 1822) & 773 & Chaetodon melannotus (Bloch \& Schneider, 1801) \\
\hline Family & Triglidae (sea robins) & 774 & Chaetodon octofasciatus Bloch, 1787 \\
\hline 731 & Chelidonichthys capensis (Cuvier 1829). & 775 & Chaetodon oxycephalus (Bleeker, 1853) \\
\hline 732 & Enneapterygius abeli (Klausewitz, 1960) & 776 & Chaetodon plebeius Cuvier, 1831 \\
\hline 733 & Helcogramma obtusirostris (Klunzinger, 1871) & 777 & Chaetodon semilarvatus Cuvier, 1831 \\
\hline 734 & Helcogramma ellioti (Herre, 1944) & 778 & Chaetodon speculum Cuvier, 1831 \\
\hline 735 & Lepidotrigla riggsi Richards \& Saksena, 1977 & 779 & Chaetodon trifascialis (Quoy \& Gaimard, 1825) \\
\hline 736 & Pterygotrigla hemisticta (Temminck \& Schlegel, 1843) & 780 & Chaetodon trifasciatus Park, 1797 \\
\hline Family & Mullidae (goatfishes) & 781 & Chaetodon vagabundus Linnaeus, 1758 \\
\hline 737 & Mullus auriflamma (Forsskål, 1775) & 782 & Chaetodon xanthocephalus Bennett, 1833 \\
\hline 738 & Mullus chrysopleuron Temmnick \& Schlegel, 1843 & 783 & Heniochus acuminatus (Linnaeus, 1758) \\
\hline 739 & Mullus barberinus (Lacepède, 1801) & 784 & Heniochus chrysostomus (Cuvier, 1831) \\
\hline 740 & Mulloidichthys flavolineatus (Lacepède, 1801) & 785 & Heniochus monoceros Cuvier, 1831 \\
\hline 741 & Parupenens pleurospilus (Bleeker) & 786 & Heniochus pleurotaenia Ahl, 1923 \\
\hline 742 & Parupeneus indicus (Shaw, 1803) & 787 & Heniochus singularius Smith \& Radcliffe, 1911 \\
\hline 743 & Parupeneus bifasciatus (Lacepède, 1801) & 788 & Heniochus varius (Cuvier, 1829) \\
\hline 744 & Parupeneus cyclostomus (Lacepède, 1801) & 789 & Megaprotodon striangulus Cuvier, 1831 \\
\hline 745 & Upeneus luzonius Jordan \& Seale, 1907 & Family & Pomacanthidae (angelfishes) \\
\hline 746 & Upeneus bensasi (Temmnick \& Schlegel, 1843) & 790 & Apolemichthys xanthurus (Bennet, 1833) \\
\hline 747 & Upeneus moluccensis (Bleeker, 1855) & 791 & Centropyge flavipectoralis Randall \& Klausewitz, 1977 \\
\hline 748 & Upeneus oligospilus Lachner, 1954 & 792 & Chaetodontoplus melanosoma (Bleeker, 1853) \\
\hline 749 & Upeneus sulphureus Cuvier, 1829 & 793 & Chaetodontoplus mesoleucus (Bloch, 1787) \\
\hline 750 & Upeneus sundaicus (Bleeker, 1855) & 794 & Pomacanthus annularis (Bloch, 1787) \\
\hline 751 & Upeneus taeniopterus Cuvier, 1829 & 795 & Pomacanthus imperator (Bloch, 1787) \\
\hline 752 & Upeneus tragula Richardson, 1846 & 796 & Pomacanthus semicirculatus (Cuvier, 1831) \\
\hline 753 & Upeneus vittatus (Forsskål, 1775) & Family & Terapontidae (grunters or tigerperches) \\
\hline Family & Pempheridae (sweepers) & 797 & Terapon jarbua (Forsskål, 1775) \\
\hline 754 & Pempheris moluca Cuvier, 1829 & 798 & Terapon theraps Cuvier, 1829 \\
\hline 755 & Pempheris vanicolensis Cuvier, 1831 & 799 & Pelates quadrilineatus (Bloch, 1790) \\
\hline 756 & Pempheris oualensis Cuvier, 1831 & 800 & Terapon puta Cuvier, 1829 \\
\hline Family & Monodactylidae (moonies) & Family & Cichlidae (cichlids) \\
\hline 757 & Monodacty/us argenteus (Linnaeus, 1758) & 801 & Etroplus suratensis (Bloch, 1790) \\
\hline Family & Kyphosidae (sea chubs) & 802 & Tilapia mossambica (Peters, 1852) \\
\hline 758 & Kyphosus cinerascens (Forsskål, 1775) & 803 & Etroplus maculatus (Bloch, 1795) \\
\hline 759 & Kyphosus vaigiensis (Quoy \& Gaimard, 1825) & Family & Pomacentridae (damselfishes) \\
\hline Family & Drepaneidae (sicklefishes) & 804 & Abudefduf bengalensis (Bloch, 1787) \\
\hline 760 & Drepane punctata (Linnaeus, 1758) & 805 & Abudefduf biocellatus (Quoy \& Gaimard 1825) \\
\hline 761 & Drepane longimana (Bloch \& Schneider, 1801) & 806 & Abudefduf notatus (Day, 1870) \\
\hline Family & Chaetodontidae (butterflyfishes) & 807 & Abudefduf septemfasciatus (Cuvier, 1830) \\
\hline 762 & Chaetodon auriga Forsskål, 1775 & 808 & Abudefduf saxatilis (Linneaus, 1758) \\
\hline 763 & Chaetodon bennetti Cuvier, 1831 & 809 & Abudefduf saxfasciatus (Lacepède, 1801) \\
\hline 764 & Chaetodon citrinellus Cuvier, 1831 & 810 & Abudefduf sordidus (Forsskål, 1775) \\
\hline 765 & Chaetodon collare Bloch, 1787 & 811 & Abudefduf uniocellatus (Quoy \& Gaimard, 1825) \\
\hline 766 & Chaetodon decussatus (Cuvier, 1829) & 812 & Abudefduf vaigiensis (Quoy \& Gaimard, 1825) \\
\hline 767 & Chaetodon fasciatus Forsskål, 1775 & 813 & Amphiprion allardi Klausewitz, 1970 \\
\hline 768 & Chaetodon gardineri Norman, 1939 & 814 & Amphiprion clarkii (Bennett, 1830) \\
\hline 769 & Chaetodon kleinii Bloch, 1790 & 815 & Amphiprion ocellaris (Cuvier, 1830) \\
\hline 770 & Chaetodon leucopleura Playfair, 1867 & 816 & Amphiprion percula (Lacepède, 1802) \\
\hline
\end{tabular}




\begin{tabular}{|c|c|}
\hline 817 & Amphiprion polymnus (Linnaeus, 1758) \\
\hline 818 & Amphiprion sebae (Bleeker, 1853) \\
\hline 819 & Chromis caerulea (Cuvier, 1830) \\
\hline 820 & Chromis castanea (Cuvier, 1814) \\
\hline 821 & Chromis dimidiata (Klunzinger, 1871) \\
\hline 822 & Chromis opercularis (Günther, 1867) \\
\hline 823 & Chromis viridis (Cuvier, 1830) \\
\hline 824 & Chrysiptera unimaculata (Cuvier, 1830) \\
\hline 825 & Dascy/lus aruanus (Linnaeus, 1758) \\
\hline 826 & Dascyllus trimaculatus (Rüppell, 1829) \\
\hline 827 & Neopomacentrus filamentosus (Macleay, 1882) \\
\hline 828 & Neopomacentrus nemurus (Bleeker, 1857) \\
\hline 829 & Plectrog/yphidodon dickii (Lienard, 1839) \\
\hline 830 & Pomacentrus trilineatus Cuvier, 1830 \\
\hline 831 & Pomacentrus tripunctatus Cuvier, 1830 \\
\hline 832 & Pomacentrus caeruleus Quoy \& Gaimard, 1825 \\
\hline 833 & Pomacentrus pavo (Bloch, 1787) \\
\hline 834 & Pomacentrus vaiuli (Jordan \& Seale, 1906) \\
\hline 835 & Premnas biaculeatus (Bloch, 1790) \\
\hline 836 & Simochromis diagramma (Günther, 1894) \\
\hline 837 & Stegastes aureus (Fowler, 1927) \\
\hline 838 & Stegastes fasciolatus (Ogilby, 1889) \\
\hline 839 & Stegastes nigricans (Lacepède, 1802) \\
\hline Family & Labridae (wrasses) \\
\hline 840 & Anampses lineatus Randall, 1972 \\
\hline 841 & Bodianus diana (Lacepède, 1801) \\
\hline 842 & Bodianus macrourus (Lacepède, 1801) \\
\hline 843 & Bodianus neilli (Day, 1867) \\
\hline 844 & Cheilio inermis (Forsskål, 1775) \\
\hline 845 & Cheilodipterus quinquelineatus Cuvier, 1828 \\
\hline 846 & Cheilinus chlorourus (Bloch, 1791) \\
\hline 847 & Cheilinus diagrammus (Lacepède, 1801) \\
\hline 848 & Cheilinus oxycephalus Bleeker, 1853 \\
\hline 849 & Cheilinus trilobatus Lacepède, 1801 \\
\hline 850 & Cheilinus undulates Rüppell, 1835 \\
\hline 851 & Coris caudimacula (Quoy \& Gaimard, 1834) \\
\hline 852 & Coris formosa (Bennet, 1830) \\
\hline 853 & Coris gaimard (Quoy \& Gaimard, 1824) \\
\hline 854 & Epibulus insidiator (Pallas, 1770) \\
\hline 855 & Gomphosus caeruleus Lacepède, 1801 \\
\hline 856 & Halichoeres zeylonius (Bennett, 1833) \\
\hline 857 & Halichoeres centiquadrus (Lacepède, 1801) \\
\hline 858 & Halichoeres hartzfeldii (Bleeker, 1852) \\
\hline 859 & Halichoeres hortulanus (Lacepède, 1801) \\
\hline 860 & Halichoeres leucurus (Walbaum, 1792) \\
\hline 861 & Halichoeres melanochir Fowler \& Bean, 1928 \\
\hline 862 & Halichoeres nigrescens (Bloch \& Schneider, 1801) \\
\hline 863 & Hemigymnus fasciatus (Bloch, 1792) \\
\hline 864 & Hemigymnus melapterus (Bloch, 1791) \\
\hline 865 & Hologymnosus annulatus (Lacepède, 1801) \\
\hline
\end{tabular}

\begin{tabular}{|c|c|}
\hline 866 & Iniistius bimaculatus (Rüppell, 1829) \\
\hline 867 & Iniistius dea (Temminck \& Schlegel, 1845) \\
\hline 868 & Iniistius pavo (Valenciennes, 1840) \\
\hline 869 & Labroides dimidiatus (Valenciennes, 1839) \\
\hline 870 & Oxycheilinus bimaculatus (Valenciennes, 1840) \\
\hline 871 & Oxycheilinus digramma (Lacepède, 1801) \\
\hline 872 & Stethojulis axillaris (Quoy \& Gaimard, 1824) \\
\hline 873 & Stethojulis balteata (Quoy \& Gaimard, 1824) \\
\hline 874 & Stethojulis interrupta (Bleeker, 1851) \\
\hline 875 & Stethojulis trilineata (Bloch \& Schneider, 1801) \\
\hline 876 & Thalassoma hardwickii (Bennett, 1830) \\
\hline 877 & Thalassoma jansenii (Bleeker, 1856) \\
\hline 878 & Thalassoma lunare (Linnaeus, 1758) \\
\hline 879 & Thalassoma purpureum (Forsskål, 1775) \\
\hline 880 & Thalassoma trilobatum (Lacepède, 1801) \\
\hline 881 & Xanthichthys ringens (Linnaeus, 1758) \\
\hline 882 & Xyrichtys margaritatus Fourmanoir, 1967 \\
\hline 883 & Xyrichtys novacula (Linnaeus, 1758) \\
\hline Family & Scaridae (parrotfishes) \\
\hline 884 & Bolbometopon muricatum (Valenciennes, 1840) \\
\hline 885 & Calotomus spinidens (Quoy \& Gaimard, 1824) \\
\hline 886 & Cetoscarus bicolor (Rüppell, 1829) \\
\hline 887 & Chlorurus gibbus (Rüppell, 1829) \\
\hline 888 & Chlorurus oedema (Snyder, 1909) \\
\hline 889 & Chlorurus sordidus (Forsskål, 1775) \\
\hline 890 & Hipposcarus harid (Forsskål, 1775) \\
\hline 891 & Leptoscarus vaigiensis (Quoy \& Gaimard, 1824) \\
\hline 892 & Scarus arabicus (Steindachner, 1902) \\
\hline 893 & Scarus denticulatus Lacepède, 1802 \\
\hline 894 & Scarus ferrugineus Forsskal, 1775 \\
\hline 895 & Scarus forsskae/ Lacepède, 1802 \\
\hline 896 & Scarus ghobban Forsskål, 1775 \\
\hline 897 & Scarus globiceps Valenciennes, 1840 \\
\hline 898 & Scarus niger Forsskål, 1775 \\
\hline 899 & Scarus psittacus Forsskål, 1775 \\
\hline 900 & Scarus rubroviolaceus Bleeker, 1847 \\
\hline 901 & Scarus russellii Valenciennes, 1840 \\
\hline 902 & Scarus tricolor Bleeker, 1847 \\
\hline Family & Ephippidae (spadefishes) \\
\hline 903 & Ephippus orbis (Bloch, 1787) \\
\hline 904 & Platax pinnatus Linnaeus, 1758 \\
\hline 905 & Platax orbicluaris (Forsskål, 1775) \\
\hline 906 & Platax teira (Forsskål, 1775) \\
\hline Family & Scatophagidae (scats) \\
\hline 907 & Scatophagus argus (Linnaeus, 1766) \\
\hline Family & Percophidae (duckbills) \\
\hline 908 & Bembrops caudimacula Steindachner, 1876 \\
\hline 909 & Bembrops platyrhynchus (Alcock, 1894) \\
\hline 910 & Bembrops curvatura Okada \& Suzuki, 1952 \\
\hline 911 & Chrionena chlorotaenia McKay, 1971 \\
\hline
\end{tabular}




\begin{tabular}{|c|c|}
\hline Family & Siganidae (rabbitfishes) \\
\hline 912 & Siganus argenteus (Quoy \& Gaimard, 1825) \\
\hline 913 & Siganus canaliculatus (Park, 1797) \\
\hline 914 & Siganus corrallinus (Valenciennes, 1835) \\
\hline 915 & Siganus javus (Linnaeus, 1766) \\
\hline 916 & Siganus lineatus (Valenciennes, 1825) \\
\hline 917 & Siganus /uridis (Rüppell, 1829) \\
\hline 918 & Siganus puellus (Schlegel, 1852) \\
\hline 919 & Siganus punctatus (Schneider \& Foster, 1801) \\
\hline 920 & Siganus spinus (Linnaeus, 1758) \\
\hline 921 & Siganus stellatus (Forsskål, 1775) \\
\hline 922 & Siganus vermiculatus (Valenciennes, 1835) \\
\hline 923 & Siganus virgatus (Valenciennes, 1835) \\
\hline Family & Cepolidae (bandfishes) \\
\hline 924 & Acanthocepola indica (Day, 1888) \\
\hline 925 & Acanthocepola limbata (Valenciennes, 1835) \\
\hline 926 & Cepola abbreviata (Valenciennes, 1835) \\
\hline 927 & Cepola coecula Bloch \& Schneider, 1801 \\
\hline Family & Zanclidae (moorish idols) \\
\hline 928 & Zanclus cronutus (Linneaus, 1758) \\
\hline Family & Acanthuridae (surgeonfishes, unicornfishes) \\
\hline 929 & Acanthaluteres spilomelanurus (Quoy \& Gaimard, 1825) \\
\hline 930 & Acanthurus annularis (Valenciennes, 1835) \\
\hline 931 & Acanthurus bariene Lesson, 1831 \\
\hline 932 & Acanthurus gahhm (Forsskål, 1775) \\
\hline 933 & Acanthurus leucosternon (Bennett, 1833) \\
\hline 934 & Acanthurus lineatus (Linnaeus, 1758) \\
\hline 935 & Acanthurus mata (Cuvier, 1829) \\
\hline 936 & Acanthurus nigricans (Linnaeus, 1758) \\
\hline 937 & Acanthurus pyroferus Kittlitz, 1834 \\
\hline 938 & Acanthurus triostegus (Linnaeus, 1758) \\
\hline 939 & Acanthurus xanthopterus Valenciennes, 1835 \\
\hline 940 & Ctenochaetus striatus (Quoy \& Gaimard, 1825) \\
\hline 941 & Naso brevirostris (Cuvier, 1829) \\
\hline 942 & Naso hexanthus (Bleeker, 1855) \\
\hline 943 & Naso lituratus (Forsskål, 1801) \\
\hline 944 & Naso tuberosus Lacepède, 1802 \\
\hline 945 & Naso unicornis (Forsskål, 1775) \\
\hline 946 & Paracanthurus hepatus (Linnaeus, 1766) \\
\hline 947 & Zebrasoma velifer (Bloch, 1795) \\
\hline 948 & Zebrasoma xanthurum (Blyth,1852) \\
\hline Family & Sphyraenidae (barracudas) \\
\hline 949 & Sphyraena barracuda (Edwards, 1771) \\
\hline 950 & Sphyraena flavicauda Rüppell, 1838 \\
\hline 951 & Sphyraena forsteri Cuvier, 1829 \\
\hline 952 & Sphyraena jello Cuvier, 1829 \\
\hline 953 & Sphyraena obtusata Cuvier, 1829 \\
\hline Family & Gempylidae (snake mackerels) \\
\hline
\end{tabular}

\begin{tabular}{|c|c|}
\hline 954 & Gempylus serpens Cuvier, 1829 \\
\hline 955 & Lepidocybium flavobrunneum (Smith, 1843) \\
\hline 956 & Neoepinnula orientalis (Gilchrist \& von Bonde, 1924) \\
\hline 957 & Rexea bengalensis (Alcock, 1894) \\
\hline 958 & Rexea promethoides (Bleeker, 1856) \\
\hline 959 & Ruvettus pretiosus Cocco, 1833 \\
\hline 960 & Thyrsites atun (Euphrasen, 1791) \\
\hline 961 & Thyrsitoides marleyi Fowler, 1929 \\
\hline Family & Trichiuridae (ribbonfishes) \\
\hline 962 & Eupleurogrammus muticus (Gray, 1831) \\
\hline 963 & Lepturacanthus savala (Cuvier, 1829) \\
\hline 964 & Trichiurus lepturus Linnaeus, 1758 \\
\hline 965 & Trichiurus auriga Klunzinger, 1884 \\
\hline Family & Scombridae (mackerels, tunas) \\
\hline 966 & Acanthocybium solandri (Cuvier, 1831) \\
\hline 967 & Auxis rochei (Risso, 1810) \\
\hline 968 & Auxis thazard (Lacepède, 1800) \\
\hline 969 & Euthynnus affinis (Cantor, 1849) \\
\hline 970 & Katsuwonus pelamis (Linnaeus, 1758) \\
\hline 971 & Rastrelliger kanagurta (Cuvier, 1816) \\
\hline 972 & Sarda orientalis (Temminck \& Shlegel, 1844) \\
\hline 973 & Scomberomorus commerson (Lacepède, 1800) \\
\hline 974 & Scomberomorus guttatus (Bloch \&Schneider, 1801) \\
\hline 975 & Scomberomorus lineolatus (Cuvier, 1831) \\
\hline 976 & Thunnus alalunga (Bonnaterre, 1788) \\
\hline 977 & Thunnus albcares (Bonnaterre, 1788) \\
\hline 978 & Thunnus obesus (Lowe, 1839) \\
\hline 979 & Thunnus tonggol (Bleeker, 1790) \\
\hline Family & Xiphiidae (swordfish) \\
\hline 980 & Xiphias gladius Linnaeus, 1758 \\
\hline Family & Istiophoridae (billfishes) \\
\hline 981 & Istiophorus platypterus (Shaw, 1792) \\
\hline 982 & Istiompax indica (Cuvier, 1832)1 \\
\hline 983 & Makaira nigricans Lacepède, 1802 \\
\hline 984 & Tetrapturus angustirostris Tanaka, 1915 \\
\hline 985 & Tetrapturus audax (Phillipi, 1887) \\
\hline Family & Ariommatidae (arriommatids) \\
\hline 986 & Arioma indica (Day, 1871) \\
\hline Family & Stromateidae (butterfishes) \\
\hline 987 & Pampus argenteus (Euphrasen, 1788) \\
\hline 988 & Pampus chinensis (Euphrasen, 1788) \\
\hline Family & Nomeidae (driftfishes) \\
\hline 989 & Cubiceps pauciradiatus Gunther, 1872 \\
\hline 990 & Cubiceps whiteleggii (Waite, 1894) \\
\hline Family & Samaridae (crested flounders) \\
\hline 991 & Samaris cristatus Gray, 1831 \\
\hline 992 & Samariscus triocellatus Woods, 1960 \\
\hline 993 & Samariscus longimanus Norman, 1927 \\
\hline
\end{tabular}




\begin{tabular}{|c|c|}
\hline 994 & Samariscus huysmani Weber, 1913 \\
\hline Family & Ateleopodidae (jellynosefishes) \\
\hline 995 & Ateleopus indicus Alcock, 1891 \\
\hline 996 & ljimaia loppei Roule, 1922 \\
\hline Family & Ammodytidae (sandlances) \\
\hline 997 & Bleekeria kallolepis (Gunther, 1829) \\
\hline 998 & Bleekeria murtii Joshi, Zacharia \& Kanthan, 2012 \\
\hline Family & Blenniidae (combtooth blennies) \\
\hline 999 & Blenniella cyanostigma (Bleeker, 1849) \\
\hline 1000 & Ecsenius bicolor (Day, 1888) \\
\hline 1001 & Ecsenius frontalis (Valenciennes, 1836) \\
\hline 1002 & Entomacrodus striatus (Valenciennes, 1836) \\
\hline 1003 & Haptogenys bipunctata (Day, 1876) \\
\hline 1004 & Omobranchus elongatus (Peters, 1855) \\
\hline 1005 & Omobranchus zebra (Bleeker, 1868) \\
\hline 1006 & Petroscirtes breviceps (Valenciennes, 1836) \\
\hline 1007 & Petroscirtes variabilis Cantor, 1849 \\
\hline 1008 & Salarias fasciatus (Bloch, 1786) \\
\hline Family & Gobiidae (gobies) \\
\hline 1009 & Acentrogobius audax Smith, 1959 \\
\hline 1010 & Acentrogobius caninus (Valenciennes, 1837) \\
\hline 1011 & Acentrogobius ennorensis Menon \& Remadevi, 1980 \\
\hline 1012 & Acentrogobius janthinopterus (Bleeker, 1853) \\
\hline 1013 & Acentrogobius masoni (Day, 1873) \\
\hline 1014 & Acentrogobius nebulosus (Forsskål, 1775) \\
\hline 1015 & Amblyeleotris diagonalis Polunin \& Lubbock, 1979 \\
\hline 1016 & Amblyeleotris guttata (Fowler, 1938) \\
\hline 1017 & Amblyeleotris gymnocephala (Bleeker, 1853) \\
\hline 1018 & Amblyeleotris steinitzi (Klausewitz, 1974) \\
\hline 1019 & Amblyeleotris wheeleri (Polunin \& Lubbock, 1977) \\
\hline 1020 & Amblygobius albimaculatus (Rüppell, 1830) \\
\hline 1021 & Amblygobius decussatus (Bleeker, 1835) \\
\hline 1022 & Amblygobius phalaena (Valenciennes, 1837) \\
\hline 1023 & Amblygobius sphynx (Valenciennes, 1837) \\
\hline 1024 & Amoya madraspatensis (Day, 1868) \\
\hline 1025 & Bathygobius cotticeps (Steindachner, 1879) \\
\hline 1026 & Bathygobius fuscus (Rüppell, 1830) \\
\hline 1027 & Bathygobius ostreicola (Chaudhuri, 1916) \\
\hline 1028 & Boleophthalmus birdsongi Murdy, 1989 \\
\hline 1029 & Boleophthalmus boddarti (Pallas, 1770) \\
\hline 1030 & Callogobius hasseltii (Bleeker, 1851) \\
\hline 1031 & Gobiodon citrinus (Rüppell, 1838) \\
\hline 1032 & Gobioides broussonnetii (Lacepède, 1800 \\
\hline 1033 & Gobius bontii Bleeker, 1849 \\
\hline 1034 & Oligolepis acutipennis (Valenciennes, 1837) \\
\hline 1035 & Parachaeturichthys polynema (Bleeker, 1853) \\
\hline 1036 & Priolepis semidoliata (Valenciennes, 1837) \\
\hline 1037 & Psamnogobius biocellatus (Valenciennes, 1837) \\
\hline
\end{tabular}

\begin{tabular}{|c|c|}
\hline 1038 & Pseudogobius javanicus (Bleeker, 1856) \\
\hline Order & PLEURONECTIFORMES \\
\hline Family & Psettodidae (righteye flounders) \\
\hline 1039 & Psettodes erumei (Bloch \& Schneider, 1801) \\
\hline Family & Paralichthyidae (large-tooth flounders) \\
\hline 1040 & Pseudorhombus annulatus Norman, 1927 \\
\hline 1041 & Pseudorhombus argus Weber, 1913 \\
\hline 1042 & Pseudorhombus arsius (Hamilton, 1822) \\
\hline 1043 & Pseudorhombus dupliciocellatus Regan, 1905 \\
\hline 1044 & Pseudorhombus elevatus Ogilby, 1912 \\
\hline 1045 & Pseudorhombus javanicus (Bleeker, 1853) \\
\hline 1046 & Pseudorhombus megalops Fowler, 1934 \\
\hline 1047 & Pseudorhombus micrognathus Norman, 1927 \\
\hline 1048 & Pseudorhombus natalensis Gilchrist, 1905 \\
\hline 1049 & Pseudorhombus triocellatus (Bloch \& Schneider, 1801) \\
\hline Family & Bothidae (lefteye flounders) \\
\hline 1050 & Arnoglossus tapeinosoma (Bleeker, 1865) \\
\hline 1051 & Arnoglossus waitei Norman, 1926 \\
\hline 1052 & Bothus bleekeri Steindachner, 1861 \\
\hline 1053 & Bothus myriaster (Temminck \& Schlegel, 1846) \\
\hline 1054 & Bothus pantherinus (Rüppell, 1830) \\
\hline 1055 & Chascanopsetta megastoma Kamohara, 1936 \\
\hline 1056 & Chascanopsetta lugubris Alcock, 1894 \\
\hline 1057 & Chascanopsetta prognatha Norman, 1939 \\
\hline 1058 & Engyprosodon grandisquama (Temminck \& Schlegel, 1846) \\
\hline 1059 & Engyprosodon macrolepis (Regan, 1908) \\
\hline Family & Soleidae (soles) \\
\hline 1060 & Aesopia cornuta Kaup, 1858 \\
\hline 1061 & Brachirus annularis Fowler, 1934 \\
\hline 1062 & Brachirus orientalis (Bloch \& Schneider, 1801) \\
\hline 1063 & Brachirus pan (Hamilton, 1822) \\
\hline 1064 & Heteromycteris oculus (Alcock, 1889) \\
\hline 1065 & Pardachirus marmoratus (Lacepède, 1802) \\
\hline 1066 & Solea elongata Day, 1877 \\
\hline 1067 & Solea ovata Richardson, 1846 \\
\hline 1068 & Synaptura commersonii (Lacepède, 1802) \\
\hline 1069 & Synaptura marginata Boulenger, 1900 \\
\hline 1070 & Zebrias synapturoides (Jenkins, 1910) \\
\hline 1071 & Zebrias quagga (Kaup, 1858) \\
\hline 1072 & Zebrias zebra (Bloch, 1787) \\
\hline Family & Cynoglossidae (tounguefishes) \\
\hline 1073 & Cynoglossus are/ (Bloch \& Schneider, 1801) \\
\hline 1074 & Cynoglossus bilineatus (Lacepède, 1802) \\
\hline 1075 & Cynoglossus brachycephalus Bleeker, 1870 \\
\hline 1076 & Cynoglossus carpenteri Alcock, 1889 \\
\hline 1077 & Cynoglossus cynoglossus (Hamilton, 1822) \\
\hline 1078 & Cynoglossus dispar Day 1877 \\
\hline 1079 & Cynoglossus dubius Day 1873 \\
\hline 1080 & Cynoglossus lida (Bleeker, 1851) \\
\hline 1081 & Cynoglossus lingua Hamilton, 1822 \\
\hline
\end{tabular}




\begin{tabular}{|c|c|}
\hline 1082 & Cynoglossus microlepis (Bleeker, 1851) \\
\hline 1083 & Cynoglossus macrostomus Norman, 1928 \\
\hline 1084 & Cynoglossus puniticeps (Richardson, 1846) \\
\hline 1085 & Cynoglossus semifasciatus Day, 1877 \\
\hline 1086 & Paraplagusia bilineata (Bloch, 1787) \\
\hline 1087 & Paraplagusia blochii (Bleeker, 1851) \\
\hline 1088 & Symphurus trifasciatus (Alcock, 1894) \\
\hline 1089 & Symphurus macrophthalmns Norman, 1939 \\
\hline 1090 & Symphurus ocellatus von Bonde, 1922 \\
\hline Order & TETRAODONTIFORMES \\
\hline Family & Triacanthodidae (spikefishes) \\
\hline 1091 & Triacanthodes anomalus (Temminck \& Schlegel, 1850) \\
\hline 1092 & Triacanthodes ethiops Alcock, 1894 \\
\hline Family & Triacanthidae (tripodfishes) \\
\hline 1093 & Triacanthus biaculeatus (Bloch, 1971) \\
\hline 1094 & Triacanthus brevirostris (Temminck \& Schlegle, 1850) \\
\hline 1095 & Pseudotriacanthus strigilifer (Cantor, 1849) \\
\hline Family & Balistidae (triggerfishes) \\
\hline 1096 & Abalistes stellaris (Bloch \& Schneider, 1801) \\
\hline 1097 & Abalistes stellatus (Anonymus, 1798) \\
\hline 1098 & Balistes capistratus Shaw, 1804 \\
\hline 1099 & Balistes rotundatus Marion de proce, 1822 \\
\hline 1100 & Balistes tomentosus Linneaus, 1758 \\
\hline 1101 & Balistapus undulatus (Park, 1797) \\
\hline 1102 & Balistoides viridescens Bloch \& Schneider, 1801 \\
\hline 1103 & Balistoides conspicillum (Bloch \& Schneider, 1801) \\
\hline 1104 & Canthidermis maculata (Bloch, 1786) \\
\hline 1105 & Melichthys niger (Bloch, 1786) \\
\hline 1106 & Odonus niger (Rüppell, 1836) \\
\hline 1107 & Pseudobalistes fuscus (Bloch \& Schneider, 1801) \\
\hline 1108 & Pseudobalistes flavimarginatus (Rüppell, 1829) \\
\hline 1109 & Rhineacanthus aculeatus (Linnaeus, 1758) \\
\hline 1110 & Rhineacanthus rectangulus (Bloch \& Schneider, 1801) \\
\hline 1111 & Sufflamen bursa (Bloch \& Schneider, 1801) \\
\hline 1112 & Sufflamen chrysopterum (Bloch \& Schneider, 1801) \\
\hline 1113 & Sufflamen fraenatum (Latreille, 1804) \\
\hline Family & Monocanthidae (filefishes) \\
\hline 1114 & Acreichthys tomentosus (Linnaeus, 1758) \\
\hline 1115 & Aluterus monoceros (Linnaeus, 1758) \\
\hline 1116 & Aluterus scriptus (Osbeck, 1765) \\
\hline 1117 & Amanses scopas (Cuvier, 1829) \\
\hline 1118 & Anacanthus barbatus (Gray, 1830) \\
\hline 1119 & Cantherhines dumerilii (Hollard, 1854) \\
\hline 1120 & Cantherhines fronticinctus (Gunther, 1867) \\
\hline 1121 & Cantherhines longicaudus Hutchins \& Randall, 1982 \\
\hline 1122 & Cantherhines pardalis (Rüppell, 1837) \\
\hline 1123 & Cantherhines rapanui (de Buen, 1963) \\
\hline 1124 & Chaetodermis penicilligerus (Cuvier, 1816) \\
\hline 1125 & Oxymonacanthus longirostris (Bloch \& Schneider, 1801) \\
\hline 1126 & Paraluteres prionurus (Bleeker, 1851) \\
\hline
\end{tabular}

\begin{tabular}{|c|c|}
\hline 1127 & Paramonacanthus arabicus Hutchins, 1997 \\
\hline 1128 & Paramonacanthus choirocephalus (Bleeker, 1852) \\
\hline 1129 & Paramonacanthus japonicus (Tilesius, 1809) \\
\hline 1130 & Paramonacanthus oblongus (Temminck \& Schlegel, 1850) \\
\hline 1131 & Paramonacanthus otisensis Whitley, 1931 \\
\hline 1132 & Paramonacanthus pusillus (Rüppell,1829) \\
\hline 1133 & Paramonacanthus tricuspis (Hollard, 1854) \\
\hline 1134 & Pervagor melanocephalus (Bleeker, 1853) \\
\hline 1135 & Pseudomonacanthus peroni (Hollard, 1854) \\
\hline 1136 & Stephanolepis auratus (Castelnau, 1861) \\
\hline 1137 & Stephanolepis diaspros Fraser- Brunner, 1940 \\
\hline 1138 & Thamnaconus modestoides (Barnard,1927) \\
\hline 1139 & Thamnaconus striatus (Kotthaus, 1979) \\
\hline Family & Ostraciidae (boxfishes, cowfishes) \\
\hline 1140 & Lactoria cornuta (Linnaeus, 1758) \\
\hline 1141 & Ostracion cubicus Linnaeus, 1758 \\
\hline 1142 & Ostracion meleagris Shaw, 1796 \\
\hline 1143 & Ostracion rhinorhynchos Bleeker, 1851 \\
\hline 1144 & Ostracion nasus (Bloch, 1785) \\
\hline 1145 & Tetrosomus concatenatus (Bloch, 1785) \\
\hline 1146 & Tetrosomus reipublicae (Whitley, 1930) \\
\hline 1147 & Tetrosomus gibbosus (Linnaeus, 1758) \\
\hline Family & Tetraodontidae (puffer fishes) \\
\hline 1148 & Amblyrhynchotes honckenii (Bloch, 1785) \\
\hline 1149 & Arothron hispidus (Linnaeus, 1758) \\
\hline 1150 & Arothron immaculatus (Bloch \&Schneider, 1801) \\
\hline 1151 & Arothron leopardus (Day, 1875) \\
\hline 1152 & Arothron reticularis (Bloch \&Schneider, 1801) \\
\hline 1153 & Arothron stellatus (Anonymous, 1798) \\
\hline 1154 & Canthigaster amboinensis (Bleeker, 1864) \\
\hline 1155 & Canthigaster bennetti (Bleeker, 1854) \\
\hline 1156 & Canthigaster margaritatus (Rüppell, 1829) \\
\hline 1157 & Canthigaster solandri (Richardson, 1845) \\
\hline 1158 & Lagocephalus gloveri Abe \& Tabeta, 1983 \\
\hline 1159 & Lagocephalus guentheri Miranda Ribeiro, 1915 \\
\hline 1160 & Lagocephalus laevigatus (Linnaeus, 1766) \\
\hline 1161 & Lagocephalus lagocephalus (Linnaeus, 1758) \\
\hline 1162 & Lagocephalus spadiceus (Richardson, 1845) \\
\hline Family & Diodontidae (porcupinefishes) \\
\hline 1163 & Chilomycterus orbicularis (Bloch, 1785) \\
\hline 1164 & Chilomycterus spilosty/us Lies \& Randall, 1982 \\
\hline 1165 & Diodon holcanthus (Linnaeus, 1758) \\
\hline 1166 & Diodon hystrix Linnaeus, 1758 \\
\hline 1167 & Diodon maculifer Kaup, 1855 \\
\hline 1168 & Lophodiodon calori (Biancon, 1850) \\
\hline Family & Molidae (ocean sunfishes) \\
\hline 1169 & Masturus lanceolatus (Lienard, 1840) \\
\hline 1170 & Masturus oxyuropterus (Bleeker, 1873) \\
\hline 1171 & Mola mola (Linnaeus, 1758) \\
\hline
\end{tabular}




\begin{tabular}{ll}
\hline 1172 & Ranzania laevis (Pennant, 1776) \\
\hline Order & OPHIDIFORMES \\
Family & Ophidiidae (cusk eels) \\
\hline 1173 & Holcomycteronus pterotus (Alcock, 1890) \\
\hline 1174 & Neobythites unimaculatus (Smith \& Radcliffe, 1913) \\
\hline 1175 & Neobythites steatiticus Alcock, 1894 \\
\hline 1176 & Neobythites stefanovi Nielsen \& Uiblein, 1993 \\
\hline 1177 & Neobythites multistriatus (Nielsen \& Quero, 1991) \\
\hline Family & Carapidae (pearlfishes) \\
\hline 1178 & Carapus mourlani (Petit, 1934) \\
\hline 1179 & Encheliophis homei (Richardson, 1846) \\
\hline 1180 & Onuxodon margaritifera (Rendahl, 1921) \\
\hline 1181 & Pyramodon ventralis Smith \& Radcliffe, 1913 \\
\hline 1182 & Pyramodon lindas (Markle \& Olney, 1990) \\
\hline
\end{tabular}

Chakrabarty et al. (2008; 2010: Silverbellies); Joshi et al. (2011 : Carangidae) and Joshi et al. (2012 a: Tuna) were useful in the compilation and preparation of systematic list of fishes in the present study.

\section{Results and discussion}

The study revealed the presence of 1,182 fish species belonging to 476 genera, 144 families and 39 orders in the Gulf of Mannar Ecosystem (Table 1). Of these 628 species were collected and identified in the present study. Details of the 554 species were taken from the past studies conducted in the GOME by earlier workers (James, 1967; Murty, 1969; Thomas, 1969; Venkataramani \& Ramaiyan, 1977; Williams \& Venkataramani, 1978; James, 1985; Venkataramani et al., 1995; Abdussamad et al., 2006; Varghese et al., 2011; Varghese, 2011; Zacharia et al., 2011; Joshi et al., 2012b, Mannisseri et al., 2012; Zacharia and Kannan, 2012).

Pioneering studies on the taxonomy of Indian fishes began in the late $18^{\text {th }}$ century by European scientists and officers of the British East India Company. One of the pioneers was Bloch (1795), and his student Schneider (Bloch and Schneider, 1801), followed by Lacepede (1798-1803). In 1794, Dr. Buchanan Hamilton, Superintendent of the Botanical gardens, Calcutta, took up a study of the fishes of the Ganges, and completed after 28 years (Hamilton, 1822) which was probably the first official catalogue of Indian fishes. Hamilton was followed by Cuvier and Valenciennes (1828) and Gunther (1859). Dr. Alcock who undertook the first marine fisheries survey in India published the findings in 1869. Perhaps the most important work during this period, pertaining to the subject was that of Sir Francis Day, Surgeon Major with the British troops in Bengal, who studied the systematics of Indian fishes in depth for over 20 years. His monumental work was published in two volumes as the 'The Fishes of India: being a natural history of the fishes known to inhabit the seas and fresh waters of India' Vol. I and II (1878) and the 'The Fauna of
British India, including Ceylon and Burma' (1889). During the subsequent period of one century, a large number of fishes have been described and added to the list prepared by Day. The important works during this period with regard to the taxonomy of fishes of the Indian waters are by Munro (1955), Jones and Kumaran (1980), who published descriptions of over 600 species from Laccadives archipelago, Talwar and Kacker (1984), and the most recent compilation of Talwar and Jhingran (1991), who published descriptions of a total of 930 species of inland (fresh and brackishwater) fishes of India.

GOMBR has a coastal line of $300 \mathrm{~km}$ and $10 \mathrm{~km}$ wide with a buffer zone on either side of the coastal zone (Melkani et al., 2006). The GOMNP is under the jurisdiction of the Wildlife Protection Act of India (1972) which prevents illegal activities in the Park. The National Park was placed under the Management of Tamil Nadu Forest Department. United Nation Development Programme (UNDP) completed a GEF funded project on the conservation and sustainable use of the Gulf of Mannar Biosphere Reserve and coastal biodiversity with the cooperation of the Government of Tamil Nadu and Government of India. Thus this area now became one of the important Marine Protected Areas of the World.

Rhinopias eschmeyeri Condé, 1977 was recorded for the first time from Indian waters in the GOM (Varghese, 2011). Two specimens of this species were landed by trawlers during November, 2009. Pelagic stingray, Pteroplatytrygon violacea (Bonaparte, 1832) was recorded for the first time from the GOM of India (Zacharia et al., 2012). A female specimen measuring $91 \mathrm{~cm}$ in TL was collected from the hooks and line landings from Tuticorin on 9 th July 2009. The species might have been migrated from the populations available in the equator, along with the monsoon currents. Two populations of $P$. violacea were present in the Indian Ocean, one along the equator between Africa and Australia and another along the southeast coast of Australia. The species recorded from the Gulf of Mannar, might have dispersed or migrated either from the equator or from South Australian waters. The presence of $P$. violacea along the GOM area of the Indian coast can be attributed to the migration of this species from the equator population.

A new fish species of the genus Bleekeria were collected by trawl nets off Tuticorin of GOMBR from a depth of 20-30 $\mathrm{m}$. Bleekeria murtii characterized by 34-39 dorsal rays, 1216 anal rays, 80-108 lateral line scales, high head length (21.1-23.2 in SL), smaller eye diameter $(2.45-5.37 \mathrm{~mm})$ and high body height $(8.7-19.7 \mathrm{~mm})$. The species-specific characters, colour pattern and morphometric variations are described in detail (Joshi et al., 2012b). Ablabys binotatus (Peters, 1855) was recorded for the first time from Indian 
waters in the GOM (Varghese, 2013). One specimen of this rare species was collected from trawl landings during November, 2007. A new record on the occurrence of Neobythites multistriatus which hitherto had not been recorded from the Bay of Bengal is reported. Two specimens measuring 160 and $180 \mathrm{~mm}$ standard length were collected from the landings of a deep-sea trawler by-catch at depths of 200-250 m off the Tuticorin coast in the Gulf of Mannar (part of the Bay of Bengal) during March 2010 (Kannan et al., 2013 a). Neobythites stefanovi is recorded for the first time from Indian waters in the Gulf of Mannar (Kannan et al., 2013 b). Two specimens were landed as by-catch in trawl gear at the Tuticorin fisheries harbor, southeast coast of India, in March 2010. This is also the first record of the species outside the Red Sea, Gulf of Oman and Gulf of Aden, in the western Indian Ocean. The taxonomy of tuna species of India is an attempt to provide adequate descriptions of all known species from Indian waters, and also to sort out various issues relating to identification of species (Joshi et al., 2012 a). The description of the otolith and the photographs are of the first of its kind on the taxonomy of tuna species which shows species specific differences in morphology and structure. The results of studies on the otolith might provide information related to the taxonomy as well as other biological aspects of tuna species. Kannan et al. (2013 c; 2013 d) recorded twostripe goby (Valenciennea helsdingenii) and striated fusilier (Caesio striata) from the Gulf of Mannar which is the first record of its range extension from the known range within the Indian Ocean.

The present systematic list of 1182 species of fin fishes of the Gulf of Mannar is one of the prerequisites for the biodiversity conservation of the renewable resources which provides the livelihood for thousands of fishermen families residing in the coastal villages of GOMBR. For a meaningful study biodiversity loss, the knowledge of the natural history of the species is necessary and this inturn can be acquired by the correct identification of species. This assumes greater importance in tropical seas where, a multitude of closely related and morphologically similar species occur (Abraham et al., 2011). The role of taxonomy, species list and proper identification cannot be overlooked in the studies of population dynamics and biodiversity conservation of GOME. The present results are also a step toward in understanding the bewildering biodiversity that characterizes the tropical seas.

This checklist of fishes with scientific names organized within a classification system will enhance the information on each species and provide more information to the stakeholders. This also has enormous value as it provides basic information required for inventory, conservation and sustainable use of biodiversity of GOME. This checklist is a first step towards generating a comprehensive list of fish species from this ecosystem and will be updated regularly as new species/ distributions are recorded.

\section{Acknowledgements}

The authors thank the Director, Central Marine Fisheries Research Institute and Scientist-in-Charge of the Tuticorin Research Centre of CMFRI for providing facilities and encouragement. The online GIS program MAPTOOL, provided by SEATURTLE.ORG is also acknowledged. The authors wish to express their gratitude towards Indian Council of Agricultural Research for funding the research work.

\section{References}

Abdussamad, E. M., T. S. Balasubramanian, O. M. M. J. Mohammed Habeeb, K. Jayabalan, G. Arumugham, D. Soundararajan and K. Manickaraja. 2006. Exploited marine fishery resources of Tuticorin along the Gulf of Mannar coast. Mar. Fish. Infor. Serv. T\&E Ser., 189:1-9.

Abraham, K. J., K. K. Joshi and V. Sriramachandra Murty. 2011.Taxonomy of the fishes of the family Leiognathidae (Pisces, Teleostei) from the West coast of India. Zootaxa, 2886: 1-18

Allen, G. R. 1979. Butterfly and Angelfishes of the world. Vol.2. John Wiley \& Sons, New York, 352 pp.

Allen, G. R. 1985. FAO species catalogue. Snappers of the world. An annotated and illustrated catalogue of lutjanid species known to date. FAO Fish. Syn. 125 (6): $208 \mathrm{pp}$.

Allen, G. R. 1991. Damselfishes of the world. Mergus publishers, Germany, 271 pp.

Allen, G. R., Swainson and J. Ruse. 1997. Marine Fishes of Tropical Australia and South-east Asia. Western Austraian Museum, Periplus Edition, 292 pp.

Anderson, W. D., Jr. 1970. Revision of the genus Symphysanodon (Pisces: Lutjanidae) with descriptions of four new species. Fish. Bull.,68 (2): 325-346.

Becker, V. E. 1968. Myctophid Fishes of the World Ocean. Science Publishing, Moscow, $248 \mathrm{pp}$.

Bellwood, D. R. 1994. A phylogenetic study of the parrot fishes family scaridae (Pisces: Labroidei), with a revision of genera. Rec. West. Aust. Mus., Supplement, 20: 1-84.

Bloch, M. E. 1795. Naturgeschichte der ausländischen Fische. Berlin, 9:192 pp.

Bloch, M. E. and J. G. Schneider. 1801. M. E. Blochii, Systemalchthyologiae Iconibus cx Ilustratum. Post obitumauctoris opus inchoatumabsolvit, correxit, interpolavit Jo. Gottlob Schneider, Saxo. Berolini. Sumtibus Auctoris Impressumet Bibliopolio Sanderiano Commissum. i-Ix ,584 pp.

Carpenter, K. E. 1987. A revision of the Indo-Pacific fish family Caesionidae, with descriptions of five new species. Indo.Pac. Fish., 15: 1-56.

Carpenter, K. E. 1988. FAO Species Catalogue. Fusilier Fishes of the world. An annotated and illustrated catalogue of Caesionid species known to date. FAO Fisheries synopsis, $125(8): 75 \mathrm{pp}$.

Carpenter, K. E. and G. R. Allen. 1989. FAO Species Catalogue. Emperor Fishes and Large- eye Breams of the world (Family Lethrinidae). An annotated and illustrated catalogue of lethrinid species known to date. FAO Fish. Syn., 125(9): 118 pp.

Carpenter, K. E. and V. E. Niem. 1998. FAO Identification Guide for Fishery Purposes. The Living Marine Resources of the Western Central Pacific Vol.2.Cephalopods, Crustaceans, Holothurians and Sharks. FAO, Rome, 1396 pp.

Chakrabarty, P. and J. S. Sparks. 2007. Phylogeny and taxonomic revision of Nuchequula Whitley, 1932 (Teleostei: Leiognathidae), with the description of a new species. Am. Mus. Novit., 3588: $1-28$.

Chakrabarty, P. and J. S. Sparks. 2008. Diagnoses for Leiognathidae Lacepède, 1802, Equula Cuvier, 1815, Equulites Fowler 1904, Eubleekeria Fowler, 1904 and a new ponyfish genus (Teleostei: Leiognathidae). Am. Mus. Novit., 3623: 1 - 11.

Chakrabarty, P., T. Amarasinghe and J. S. Sparks. 2008. Redescriptions of ponyfishes (Teleostei: Leiognathidae) of Sri Lanka and the status of Aurigequula Fowler, 1918. Ceyl. Jour. Sci. (Biological Science), 37(2): 143 - 161.

Chakrabarty, P., J. Chu, N. Luthfun and J. S. Sparks.2010. Geometric morphometrics uncovers an undescribed ponyfish (Teleostei: Leiognathidae: Equulites) with a note on the taxonomic status of Equula berbis Valenciennes. Zootaxa, 2427: $15-24$.

Chen, H. M., K. T. Shao, and J. T. Chen. 1994. A review of the muraenid eels (family Muraenidae) from Taiwan with description of twelve new records. Zool. Stud. (Taiwan), 34 (3): 202-205. 
Choat, J. H. and J. E. Randall. 1986. A review of the parrotfishes (family Scaridae) of the Great Barrier Reef of Australia with description of a new species. Reco. Aust. Mus., 38: 175-228.

Collette, B. B. and C. E. Nauen. 1983. FAO Species Catalogue.Scombrids of the world. An annotated and illustrated catalogue of tunas, mackerels, bonitos and related species known to date. FAO Fish. Syn., 125 (2) : 137 pp.

Cressey, R. 1981. Revision of Indo-west Pacific lizards of the genus Synodus (Pisces: Synodontidae). Smith. Cont. Zool., 342, 53 pp.

Cuvier, G. and A. Valenciennes. 1828. Histoire naturelle des poissons.Tome second. Livre Troisième. Des poissons de la famille des perches, ou des percoïdes. Hist. Natur. despois., 2, $490 \mathrm{pp}$

Dawson, C. E. 1985. Indo-Pacific Pipefishes (Red Sea to the Americas). The Gulf Coast Research Laboratory, Ocean Springs, Mississippi, USA, 230 pp.

Day, F. 1878. The fishes of India; being the natural history of the fishes known to inhabit the seas and fresh waters of India, Burma and Ceylon. London, 1: 729 pp.

Day, F. 1888. The fishes of India; being a natural history of the fishes known to inhabit the seas and fresh waters of India, Burma, and Ceylon. Fishes India, Suppl., $816 \mathrm{pp}$.

Day, F. 1889. Fishes. In: W. T. Blanford (ed.) The fauna of British India, including Ceylon and Burma. Taylor \& Francis, London. v. 2: 509 pp.

Devanesan and Chidambaram. 1956. Results obtained at the pearl oyster farm, Krusadai Island Gulf of Mannar and their application to problems relating to the pearl fisheries in the Gulf of Mannar - Part-I. Contributions to Marine Fishery Biology Station Krusadai Island Gulf of Mannar, 4: 1-89.

Dooley, J. K. 1978. Systematics and biology of the tilefishes (Perciformes: Branchiostegidae and Malacanthidae) with descriptions of two new species. NOAA Tech. Rep., NMFS Circular, 411: 1-78.

Eschmeyer, W. N. and R. Fricke 2015. Catalogue of fishes; genera, species, references. Electronic version accessed 2015

Eschmeyer, W. N. 1998. Catalog of Fishes. Center for Biodiversity Research and Information, Special Publication 1. California Academy of Sciences. vols. 1-3: $2905 \mathrm{pp}$.

Fischer, W. and P. J. P. Whitehead. 1974. FAO species identification sheets for fishery purposes. Eastern Indian Ocean and Western Central Pacific (Fishing areas 57 and 71), FAO, Rome.

Froese, R. and D. Pauly. 2015. Fishbase. World Wide Web Electronic Publication.www. fishbase.org.

Gon, 0. 1993. Revision of the Cardinalfish genus Cheilodipterus (Perciformes: Apogonidae), with description of five new species. Ind. Pac.Fish., 22: 1-59.

Gunther, A. 1859-1870. Catalogue of the Fishes of the British Museum. In 8 volumes.

Hamilton, F. 1822. An account of the fishes found in river Ganges and its branches. Edinberg, $405 \mathrm{pp}$

Heemstra, P. C. and J. E. Randall. 1993. FAO Species Catalogue. Groupers of the world (Family Serranidae, Subfamily Epinephelinae).An annotated and illustrated catalogue of the grouper, rockcod, hind, coral grouper and lyretail species known to date. FAO Fish. Syn., 125(16): 382 pp.

Heemstra, P. C. 1980. A revision of the zeid fishes (Zeiformes: Zeidae) of South Africa. Ichthyo. Bull. J. L. B. Smith Inst. Ichth., 41: 1-18.

Herdman, W. A. 1903-1906. Report to the Government of Ceylon on the Pearl oyster Fisheries of Gulf of Mannar.Royal Society, London, 1: 384pp, 4: 326 pp.

Hoese and Larson. 1994. Revision of the Indo-Pacific gobiid fish genus Valenciennea, with descriptions of seven new species. Ind. Pac. Fish., 23: 1-71.

Hornell, J. 1916. An explanation of the cyclic characters of the pearl fisheries of Gulf of Mannar. Madras Fish. Bull., 8: 11-22.

Hornell, J. 1922. The Indian pearl fisheries of the Gulf of Mannar. Mad. Fish. Bull.,16:1-188.

Imamura, H. 1996. Phylogeny of the family Platycephalidae and related taxa (Pisces: Scorpaeniformes). Spec. diver., 1(2): 123-233.

James, P. S. B. R. 1967. MBAI Memoir No. 1: The ribbon-fishes of the family Trichiuridae of India, Technical Report. Marine Biological Association of India, Mandapam Camp. 245 pp.

James, P. S. B. R. 1985. Comparative Osteology of the fishes of the family Leiognathidae Part II: Relationships among the genera and the species. Indian J. Fish., 32 (4): 395-416.

Jones. S and M. Kumaran. 1980. Fishes of the Laccadive Archipelago. Mathrubhumi press, Cochin, $676 \mathrm{pp}$

Joshi, K. K., Rekha J. Nair, E. M. A. Samad, Sujitha Thomas, V. S. Kakati, S. Jasmine, Molly Varghese, Miriam Paul S, Sandhya Sukumaran, Rani Mary George and Mary K. Manisseri. 2011. The Carangids of India - A Monograph. Central Marine Fisheries Research Institute, Kochi, 437 pp.

Joshi, K. K., E. M., Abdussamad, K. P. Said Koya, Prathibha Rohit, Shubhadeep Ghosh, K. R. Sreenath, M. Beni, K. K. Bineesh and K. V. Akhilesh. 2012 a. Taxonomy and key for the identification of tuna species exploited from the Indian EEZ. Indian $\mathrm{J}$. Fish., 59(3): 53-60.

Joshi, K. K., P. U. Zacharia and K. P. Kanthan. 2012 b. Description of a new sand lance species, Bleekeria murtii (Perciformes: Ammodytidae) from India. Indian J. Fish., 59 (2): 101-107.
Kannan, K., K. K. Joshi, S. John,Sand J. A. Johnson. 2013a. New record of Neobythites multistriatus (Pisces: Ophidiidae) from Bay of Bengal, south-east coast of India. Mar. Biod. Reco., 6, e83, pp 1-3, doi: 10.1017/S1755267213000572.

Kannan, K., S. John, K. K. Joshi and P. U. Zacharia. 2013b. First record of Neobythites stefanovi (Teleostei: Ophidiidae) from Indian waters. Mar. Biod. Reco. 6, e71, pp 1-3, doi: 10. 1017/S1755267213000523.

Kannan, K., Sajan John, J. A. Johnson, P. U. Zacharia and K. K. Joshi. 2013 c. First record of striated fusilier Caesio striata (Teleostei: Caesionidae), from Indian waters. Mar. Biod. Reco., 6 (106). pp. 1-3.

Kannan, K. , K. Suresh Kumar, L. Ranjith, K. K. Joshi, M. S. Madan and Sajan John. 2013d. First record of the two stripe goby, Valenciennea helsdingenii (Gobiidae, Gobiiformes) from the southeast coast of India. Zoo Keys, 323. pp. 91-97.

Katayama, M. 1960. Fauna japonica. Serranidae (Pisces). Biogeographical Society of Japan, Tokyo, $189 \mathrm{pp}$

Kawaguchi, K. and H. Shimizu. 1978. Taxonomy and distribution of the lantern fishes, genus Diaphus (Pisces, Myctophidae) in the western Pacific, eastern Indian Ocean and Southeast Asian Seas. Bull. Ocean Res. Inst., University of Tokyo, 10: 1-145.

Kishimoto, H., M. Hayashi, H. Kohno and O. Moriyama. 1988. Revision of the Japanese batfishes, genus Platax. Sci. Rep. Yoko. City Museum, 36: 19-38.

Knapp, L. W. 1996. Review of the genus Cociella Whitley (Teleostei: Platycephalidae) with the description of three new species. Proc. Biol. Soc. Washington, 109 (1): 17-23.

Kotlyar, A. N. 1987. Classification and distribution of fishes of the family Diretmidae (Bercyiformes). Vopr. Iktiol., 27 (6): 883-897.

Kotlyar, A. N. 1996. Beryciform Fishes of the World Ocean. VNIRO Publishing, Moscow, $367 \mathrm{pp}$.

Lal Mohan, R. S. 1981. An illustrated synopsis of the fishes of the family Sciaenidae of India. Indian J. Fish., 28 (1\&2): 1-24.

Lacepede, B. G. E. V. 1798-1803. Histoire naturalle des Poissonsdedice au citoyen. Lacepede.Vols.I to V. Histoire naturelle des poissons.Tome cinquième. Chez Plassan, Paris, p. 1-532

Leis, J. M. 1978. Systematics and Zoogeography of the porcupine-fishes (Diodon, Diodontidae, Tetraodontiformes) with comments on egg and larval development. $U$. S. Fish. Bull., 76 (3): 535-567.

Lin, L. H. and K. T. Shao. 1987. Fishes of the family Sphyraenidae of Taiwan. J. Taiwan Mus., 40 (2): $73-89$.

Lourie, S. A., A. C. J. Vincent and H. J. Hall. 1999. Seahorse: An identification guide to the world's species and their conservation. Project Seahorse, London, U. K. 214 pp.

Manisseri, Mary K., Geetha Antony, Rani Mary George, Rekha J Nair, K. K. Joshi and P. M. Geetha. 2012. Marine Biodiversity Museum (A Designated National Repository) Catalogue. CMFRI Special Publication, 107. Central Marine Fisheries Research Institute, Kochi, $222 \mathrm{pp}$.

Markel, D. F. and J. E. Olney. 1990. Systematics of the pearl fishes (Pisces: Carapidae). Bull. Mar. Sci., 47 (2): 269-410.

McKay, R. J. 1992. FAO Species Catalogue.Sillaginid Fishes of the world (Family Sillaginidae).An annotated and illustrated catalogue of the sillago, smelt or IndoPacific whiting species known to date. FAO Fish. Syn. 125 (14), 87 pp.

Melkani,V. K., J. K. Patterson Edward, A. Murugan, Jamila Pattersen and V. Naganthan. 2006. Capacity building in Marine Biodiversity Conservation, Ramanathapuram. Gulf of Mannar Biosphere Reserve Trust, 2006.

Menon, A. G. K. 1977. A systematic monograph of the tongue soles of the genus Cynoglossus Hamilton-Buchanan (Pisces: Cynoglossidae). Smith. Contr. Zool., 238,129 pp.

Munro, I. S. R. 1955. The Marine and Freshwater Fishes of Ceylon.Canberra: Department of External Affairs, $351 \mathrm{pp}$.

Munroe, T. A. and B. N. Marsh. 1997. Taxonomic status of three nominal species of Indo-Pacific symphurine tonguefishes (Symphurus: Cynoglossidae: Pleuronectiformes). Ichthoyl. Res., 44 (2): 189-200.

Murdy, E. O. 1989. A taxonomic revision and cladistics analysis of the oxudercine gobies (Gobiidae: Oxudercinae). Rec. Aust. Mus. Supplement,1: 1-93.

Murdy, E. O. and D. F. Hoese. 1985. Revision of the gobiid fish genus Istigobius. Ind. Pac. Fish., 4: 1-41.

Murty, Sriramachandra, V. 1969. In: Catalogue of fishes (excluding from Laccadives) in the reference collections of Central Marine Fisheries Research Institute. Bull. Cent. Mar. Fish. Res. Inst., 10:36 pp.

Nakamura, I. and N. V. Parin. 1993. FAO Species Catalogue. Snake Mackerels and cutlassfishes of the world (Families Gempylidae and Trichiuridae). An annotated and illustrated catalogue of the snake mackerels, snoeks, escolars, gemfishes, sackfishes, domine, oil fish, cutlass fishes, scabbard fishes, hair tails, and frost fishes known to date. FAO Fish. Syn., 125 (15), 136 pp.

Nayar, Nagappan, K. and S. Mahadevan. 1973. Chank resources of India. In: Proceedings of the symposium on living resources of the seas around India, 1968, Mandapam Camp, 672-686.

Nelson, J. 2006. Fishes of the world. John Wiley and Sons, New York. 416 pp.

Randall, J. E. 1982. A review of the labrid fish genus Hologymnosus. Revue francaise d'Aquariologie Herpetologie, 9 (1): 13-20. 
Randall, J. E. and J. E. McCosker. 1992. Revision of the fish genus Luzonichthys (Perciformes: Serranidae: Anthiinae), with descriptions of two new species. Ind. Pac. Fish., 21: 1-21.

Randall, J. E. and P. C. Heemstra. 1991. Revision of the Indo-Pacific groupers (Perciformes: Serranidae: Epinephelinae), with descriptions of five new species. Ind. Pac. Fish., 20: 1-332

Randall, J. E. and C. C. Baldwin. 1997. Revision of the serranid fishes of the subtribe Pseudogrammina, with descriptions of five new species. Ind. Pac. Fish., 26: $1-56$.

Randall, J. E., E. A. Lachner and T. H. Fraser. 1985. A revision of the Indo-Pacific apogonid fish genus Pseudamia, with descriptions of three new species. Ind. Pac. Fish., 6: 1-23.

Randall, J. E., G. R. Allen and W. D. Anderson, Jr. 1987. Revision of the Indo-Pacific lutjanid genus Pinwale, with description of a new species. Ind. Pac. Fish., 14: 1-17.

Randall, J. E. and D. W. Greenfield. 1996. Revision of the Indo-Pacific holocentrid fishes of the genus Myripristis, with description of three New Species. Ind. Pac. Fish., 25: 1-61.

Roberts, T. R. and C. Vidthayanon.1991.Systematic revision of the Asian catfish family Pangasidae with biological observations and descriptions of three new species. Proc. Nat. Sci. Phila., 143: 97-144.

Russell, B. C. 1990. FAO species catalogue.Nemipterid Fishes of the world (Threadfin breams, whiptail breams, monocle breams, dwarf monocle breams and coral breams). An annotated and illustrated catalogue of nemipterid species known to date. FAO Fish. Catal., 125 (12) 149 pp.

Senon, H. 1988. Phylogenetic interrelationships of the mullet (Pisces: Mugilidae). Doctoral thesis of the University of Tokyo, $172 \mathrm{pp}$.

Smith, M. M. and P. C. Heemstra. 1986. Smith's Sea Fishes of South Africa. McMillan, Johannesberg, $1047 \mathrm{pp}$.

Sparks, J. S. and P. Chakrabarty. 2007. A new species of ponyfish (Teleostei: Leiognathidae:Photoplagios) from the Philippines. Copeia, 2007 (3): 622-629.

Springer, V. G. 1971. Revision of the fish genus Ecsenius (Blennidae, Blenninae, Salariini). Smith. Contr. Zool., 72: 1-74.

Springer, V. G. and J. T. Williams. 1994. The Indo-west-Pacific blennid fish genus Istiblennius reappraised: a revision of Istiblennius, Blenniella, and Paralticus, new genus. Smith.Contr. Zool., 565,193 pp.

Starnes, W. C. 1988. Revision, phylogeny and biogeographic comments on the circumtropical marine percoid fish family Priacanthidae. Bull. Mar. Sci., 43 (2): 117-203.
Talwar, P. K. and R. K. Kacker. 1984. Commercial sea Fishes of India. Publ. by Zoological Survey of India, Calcutta, 997 pp.

Talwar, P. K. and A. G. Jhingran. 1991. Inland fishes of India and adjacent countries. In Vol-I \& II, Oxford \& IBH Publishing Co., New Delhi, Bombay, Calcutta, 1158 pp.

Thomas, P. A. 1969. MBAl Memoir No. 3: Goat fishes (Family Mullidae) of the Indian seas. Marine Biological Association of India, Cochin, 174 pp.

Thomson, J. M. 1997.The mugilidae of the world. Mem. Que. Mus., 41(3): 457-562.

Trewavas, E. 1977. The sciaenid fishes (croakers or drums) of the Indo-West-Pacific. Trans. Zool. Soci. London, 33: 253-541.

Varghese Molly. 2011. Rhinopias eschmeyeri Conde, 1977 (Family: Scorpaenidae) a new record from Indian waters. J. Mar. Biol. Ass. India, 53 (1):89-93

Varghese Molly. 2013. First record of Ablabys binotatus (Scorpaeniformes: Tetrarogidae) from Indian waters. Mar. Biod. Rec., 6. pp. 1-3.

Varghese Molly., Mary K. Manisseri, N. Ramamurty, P. M. Geetha, V. J. Thomas and A. Gandhi. 2011. Coral reef fishes of Gulf of Mannar, S.E. of India. Fishing Chimes, 31 (1): 38-40.

Vari, R. P. 1978. The Terapon perches (Percoidei, Teraponidae). A cladistics analysis and taxonomic revision. Bull. Amer. Mus. Nat. His.,159 (5): 177-340.

Venkataramani, V.K. and V. Ramaiyan. 1977. A carangid fish Caranx williamsi (Smith, 1968) (Pisces: Carangidae). Curr. Sci., 46 (1): 26 pp.

Venkataramani, V.K., N. Ramanathan, K. Venkataramanujan and V. Ramiyan. 1995. Carangid Fishes (Family: Carangidae) of Parangipettai (Porto Novo), India. Mem. CAS. Mar. Biol., Fasicle 3:83 pp.

Weber, M. and L. F. deBeaufort. 1913-1940. The fishes of the Indo-Australian Archipelago. Vol.II -X. E.J. Brill, Leiden.

Williams, F. and V.K. Venkataramani. 1978. Notes on Indo-pacific carangid fishes of the genus Carangoides Bleeker. I. The Carangoides malabaricus group. Bull. Mar. Sci. 28(3): 501-511.

Woodland, D. J. 1990. Revision of the fish family Siganidae with descriptions of two new species and comments on distribution and biology. Ind. Pac. Fish. ,19: 136.

Zacharia, P. U., K. K. Joshi and P. Kandan. 2011. First record of pelagic stingray Pteroplatyrygon violacea (Bonaparte, 1832) (Family: Dasyatidae) from the east coast of India. Indian J. Fish., 58 (1): 95-98.

Zacharia, P. U. and K. Kannan. 2012. First record of Polka-dot ribbonfish Desmodema polystictum (Pisces: Trachipteridae) from Indian waters. Mar. Biod. Reco., 5 (8): $1-4$. 